Distributiun ('ategory:

Mathematics and

('ompulter Sicienes (1)(-405)

ARGONNE NATIONAL IABORATORY

9700 Sumth e ass Avemue

Argonm, 11, 1504:29-1801

ANL $--92 / 47$

DE93 008166

ANL-92/47

\title{
A Test Implementation of the MPI Draft Message-Passing Standard
}

by

William Gropp and Euing Lusk

Mathematics and ('omputer Science. Division

December $199^{\circ}$

\section{MASTER}

This work was supported in part by the Office of Sicientific (omputing. V.L. Department of Encrgy.<smiles>C1C2C3C1C23</smiles> 


\section{Contents}

$\begin{array}{lll}\text { Abstract } & 1\end{array}$

1 Introduction 1

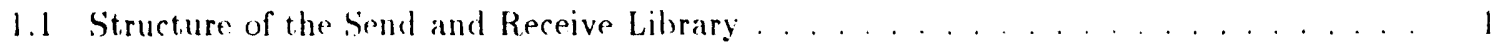

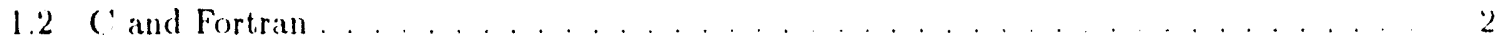

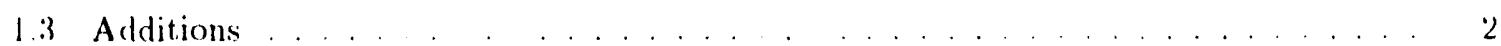

1.4 Omissions . . . . . . . . . . . . . . . . . . . . . . .

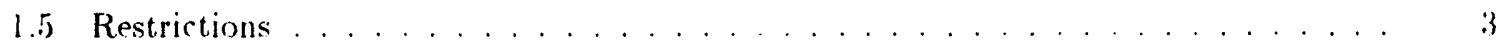

2 Comments on the Draft Standard 3

3 Starting and Stopping Processess 4

4 Very Simple User Interface $\quad 4$

5 Examples 4

6 Availability 5

7 Basic Routines for Point-to-Point Messages 5

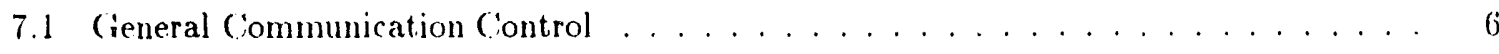

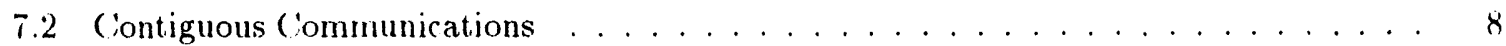

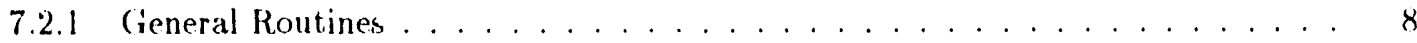

7.2 .2 Blocking Routines . . . . . . . . . . . . . . . . 13

7.2 .3 Nonblocking Routines . . . . . . . . . . . . . . . . . . 18

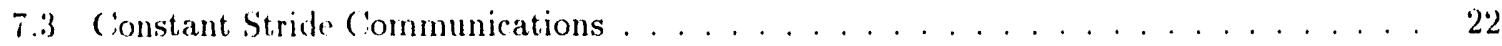

7.3 .1 (ieneral Routines . . . . . . . . . . . . . . . . . . 22

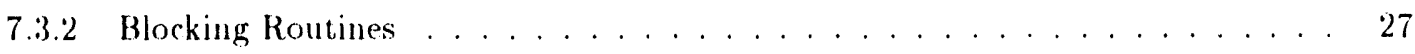

7.3 .3 Nonblocking Routines . . . . . . . . . . . . . . . . . . 32

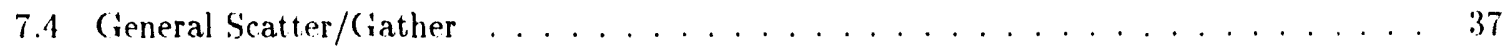

7.4 .1 General Routines . . . . . . . . . . . . . . . . 37

7.4 .2 Blocking Routines . . . . . . . . . . . . . . . . 42

7.4.3 Nonblocking Routines . . . . . . . . . . . . . . . . . . . 40

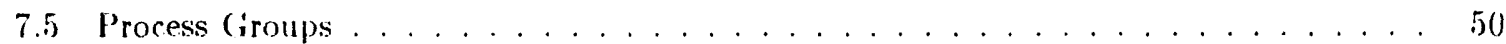

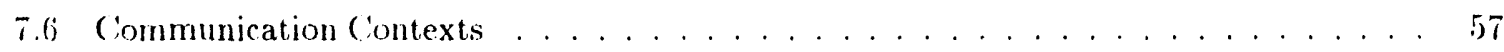

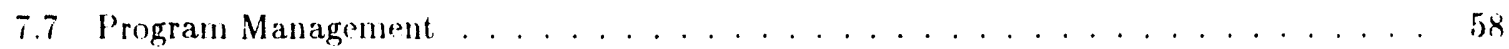

7.8 Utility Rontines. . . . . . . . . . . . . . . . . . . . . . . . 59

$\begin{array}{lll}\text { Index } & 62\end{array}$ 


\title{
A Test Implementation \\ of the MPI Draft Message-Passing Standard
}

\author{
by \\ William Gropp and Ewing Lusk
}

\begin{abstract}
Message passing is a common method for programming parallel computers. The lack of a standard has significantly impeded the development of portable software and libraries for these machines. Recently, an ad-hoc committee was formed to develop a standard for message-passing software for parallel computers. This group first met in April 1992 at a workshop sponsored in part by the (ienter for Research on Parallel (iomputation (CRP(:). Four of the attendees at that meeting produced a draft standard, henceforth referred to as the MP'I (Message- $\mathrm{F}_{\text {assing }}$ Interface) draft standard. After review by a larger group, and significant changes in the docmment, a meeting was held in November to discuss the MPI draft standard. This document is a result of those discussions; it describes a running implementation of most of the proposed standard, plus additional routines that were suggested by the discussions at the November meeting.
\end{abstract}

\section{Introduction}

This document describes a test implementation of the Message-Passing Interface (MPI) draft standard. Supplying an implementation along with the standard itself provides several benefits. It allows the draft standard to be tested for expressivity and implementability. It brings to light potential inconsistencies and omissions in the draft standard as the draft standard develops. It allows xperiments that measure possible restrictions on performance imposed by the draft standard.

Sulpject to the small number of restrictions and omissions cited below, it is an implementation of all of the draft standard. It also includes other routines that might be considered for inclusion in the draft standard as it develops.

\subsection{Structure of the Send and Receive Library}

This implementation provides a relatively large number of simple operations that are small and therefore easy to describe precisely. Larger operations can then be implemented and defined in terms of these operations. The basic send and receive operations are broken down as follows:

$$
\left[\begin{array}{l}
c \\
s \\
g
\end{array}\right]\left[\begin{array}{l}
n \\
b \\
s
\end{array}\right]\left[\begin{array}{c}
s e n d \\
r e c v
\end{array}\right]\left[\begin{array}{l}
\vec{h}
\end{array}\right]\left[\begin{array}{c}
- \\
r r
\end{array}\right],
$$

where the first letter specifies the layout of the data, the second specifies the extent to which the calling process synchronizes with the local message-passing subsystem and with the remote one, the IIext four letters specify a send or receive operation, and the final letter(s) specify optional additional functionality.

- Data layout

c (contiguous) The bytes are to be sent from or received into a contiguous region of memory, described hy starting address and length.

s (stride) The data to be sent consists of data items of the same type and size, separated by a constant distance (stride) in memory, described by a starting address, length of a single item, and number of items. 
Ir (gather) The data to be sent consists of data items of varying lengths and addresses, described by an array of addresses and lengths.

- Synchronization

n (nonblocking) The operation returns control to the user immediately, to facilitate overlappling computation and communication.

b (blocking) the operation does not return until the message area is available for reuse.

$s$ (synchronous) The operation does not return until the message has been received by the destination process (in the case of a send) or the acknowledgment has been sent (in the (ase of a receive).

- Send or Receive

send (send) Transfer a message from the calling process to the specified one.

recv (receive) Transfer a message into memory if specified conditions are met.

- Heterogeneity

- (default) This is the default.

h (heterogeneous) The message will be processed in such a way that differing data representations on different machines will be taken into account. On the $[c]$ and $[s]$ routines this requires a ditta type parameter. On the $[\mathrm{g}]$ operations, it requires data type information in the vector describing the data location.

- Alternate Protorol

- (default) This is the default.

$\mathbf{r r}$ (receiver ready) The operation will take advantage of any underlying protocol that is available when the receiver of a message is known to have issued the receive before the corresponding send is executed.

The routines specited in the current (lraft standard, which use a mode argument to specify the synchronization level, can easily be defined (and implemented) in terms of these operations, and wo have done so. This organization makes il eisy to experiment with and understand capabilities not currently in the draft, such as the $r$ and $h$ options.

\section{$1.2 \mathrm{C}$ and Fortran}

The implementation described here is for the (: language. Enough of the Fortran versions of these routiues have been provided to write and run a simple program. These are the routines MPI_csend, MPI_crecv, a few inquiry routines (e.g., MFI_getid), and MPI_main.

\subsection{Additions}

In urder to write actual programs, it was necessary to add a few routines for program management. Wir have adlited the routimes MPI main and MPI_stopall for this purpose.

We also have suggesterl a suall set of routines (see Section 4) that are at a higher level than the draft siandard, in order to monet the needs of users who wish to express a message-passing algorithu, but whor de not need to know about the more complex performance issues this implementation and the Iraft standard itself tries to address, such as overlapping communication and computation with $\mathrm{n}$ or reducing latency with $\mathrm{rr}$.

Wir also added an error valur for "moknown mode." 


\subsection{Omissions}

Wriave not implemented the time and date routines since there semed to be a consensus that they need not be part of this standard. We can add them if they are desired. We did not implement. the MPI_pack, etc., routines because they now seem to be subsumed by the [s] and [ $\mathrm{g}$ ] versions of send and receive. The man pages do not yet include the "I)escription" section; this can in most cases he taken from the draft standard.

\subsection{Restrictions}

This is a very preliminary, fast implementation, designed to allow experimentation with at least somr of the ideas in the draft standard. In the interest of getting it out very quickly, we have taken some shiurtcuts. We intend to remove these as time goes hy. ('urrently (November 25, 1992) the following restrictions apply to the implementation of the draft standard:

- There is only one process group. Since the draft standard does not specify that there be mure than one, this implenentation is (draft.) standard-conforming. However, it is not currently possible to run a program that uses more than one group. (You are likely to get the "too many groups" error.)

- There is only one communication context. Again, this conforms to the litter but not the spirit. of the draft standard.

- Neither selection on source nor selection on type range (that is, the negative type values) is implemented. This restriction allows us to use existing (vendor-supplied) message-passing implementations on a variety of machines.

\section{Comments on the Draft Standard}

One reason for doing a prototype implementation is to identify potential problems with the specification of the standard. In this section, we detail some of the problems that we have detected.

- The routines that take mode for "blocking," "nonblocking," or "synchronous" return a valur whose meaning depends on the value of mode.

- The routines to get information on "the last message" are tricky to specify precisely, because the notion of "the last message" is imprecise. In particular, "the last message" means thr last message received, probed, or otherwise looked at. This means that an MPI_probe will change the values that these routines will return. It is also unclear how process groups and communications contexts affect the meaning of "the last message."

- The standard specifies a 32 bit type field. With 64 bit systems on the horizon, this seems shortsighted.

- Because there are no minimum number of communications contexts or process groups specified, an implementation can conform to the standard by providing a single communication context. and a single process group. This is in fact what our implementation dors.

- Were process groups to be implemented, the interpretation of the destination ficld (dest) and the return valur from MPI_infos is unclear. Is it the process id? Is it the rank of the process in the current group? What is the meaning of receive-from-any-processor as a selection in a recrive routine?

- There is no way for the user to control the ranking of processes in a process gronlp. 
- There is no way to discover the length of a message before recriving it into a user buffer. 'This prohibits using dynamic memory allocation (either by malloc in ( $:$ or hy explicit allocatim if work areas in Fortran $7 i$ ) to manage messages that are of unknown length at compile timn.

- As the examples demonstrate, there is no attractive way to determine such simple things as the procens id or the number of processes.

- Frror handling is mattractive. A: written, the user must check return codes. While we anrer. that users (particularly software library writers) need this option, not all users will be diligent about checking the return codes. One of the examples below emphasizes this.

\section{$3 \quad$ Starting and Stopping Processes}

In this section we give the specifications for routines needed to support the creation and destruction of the processes that will be communicating. We have added a routine MPI_stopall that causes all processes in an application to exit.

It has been our experience that a major source of portability problems is in how a parallel progran is started up and initializes its environment. We have added MPImain as a standardized way 1.0 accomplish this. This replaces main in (" and PROGRAM in Fortran 77. In addition, it may be useful to provide a subroutine-level interface for initializing the MPI package.

\section{Very Simple User Interface}

A very simple interface can be lefined that consists of the rontines

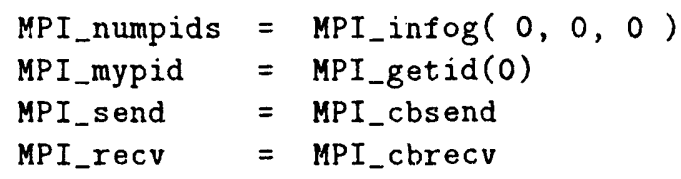

as Well as MPI main for defining a program.

\section{Examples}

We presemt here two programs that send a message around a ring of processors. The Fortran version of this progrant is

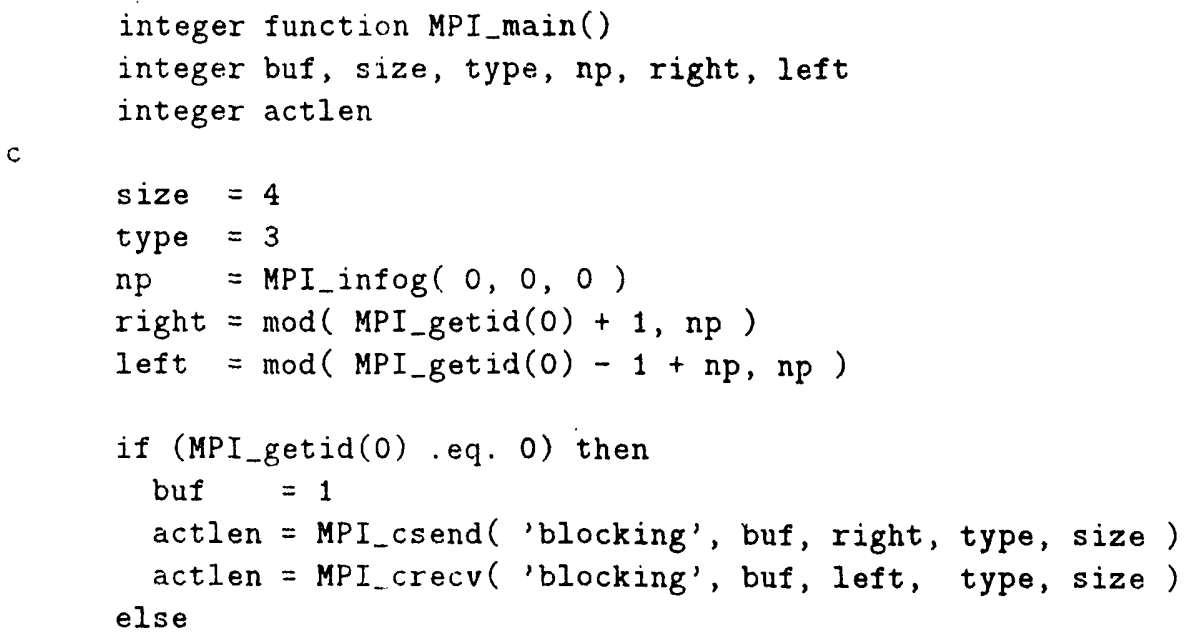




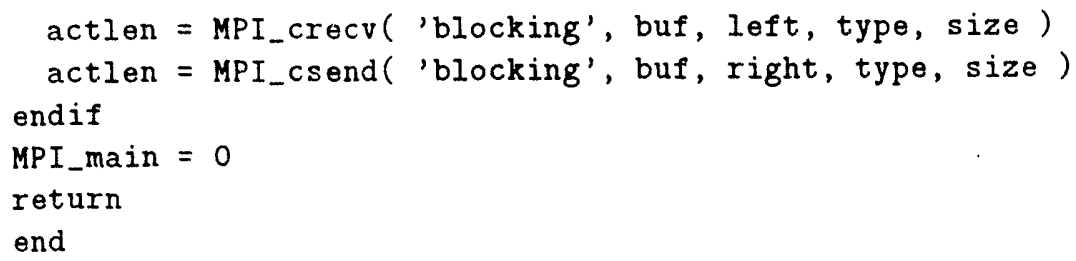

'The ("version of this program is

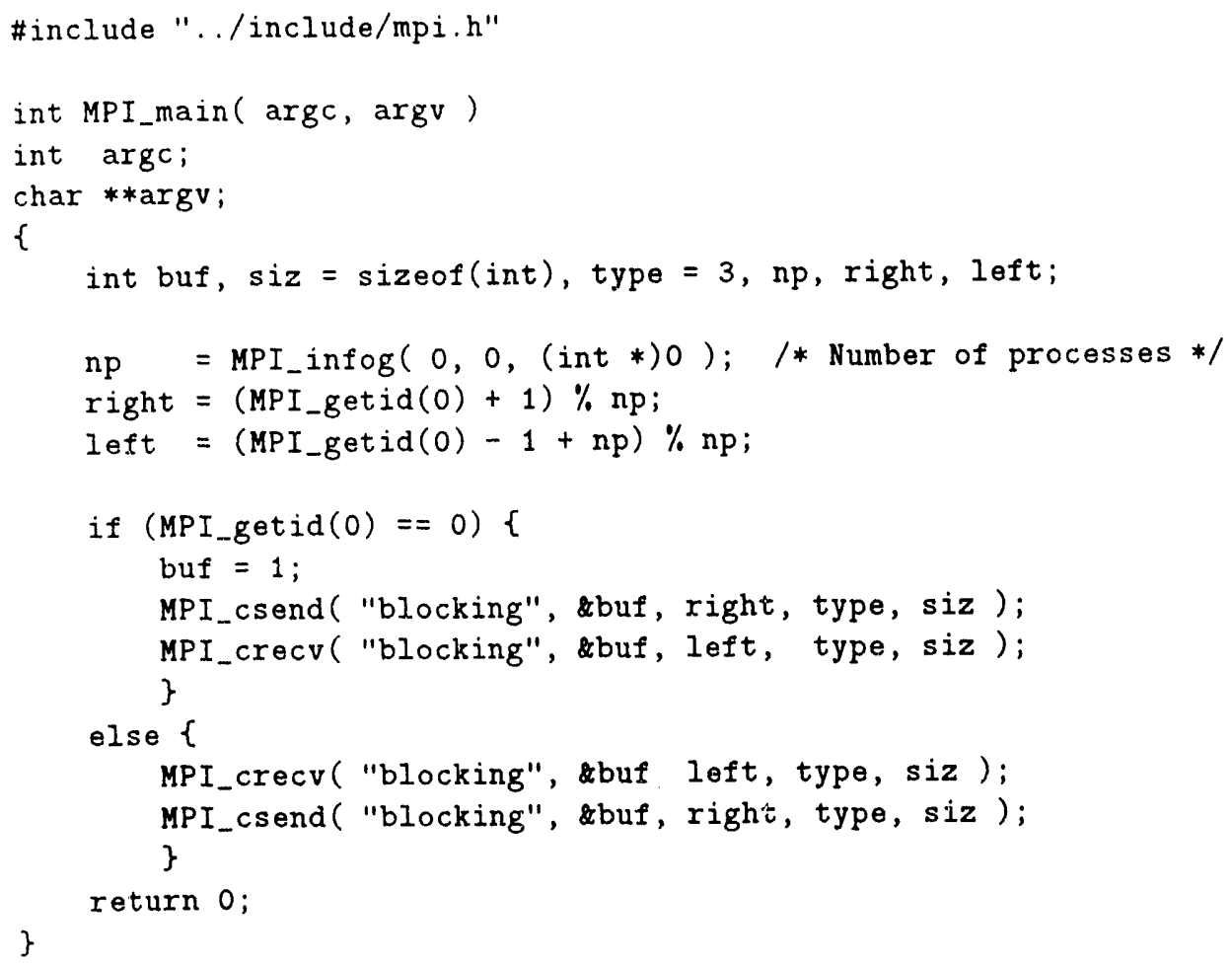

\section{Availability}

This implententation is a' ilable by anonymous ftp from info.mcs.anl.gov. In the directory pub/mpi the file mpi.man.ps. $Z$ is this document. The file mpi.tar. $Z$ contains a compressed tar file of this implementation. The implementation is built on top of the Chameleon system; the file chameleon.tar.Z is all that is needed to build ('hameleon for a variety of parallel computers (including groups of workstations). To use MPI on a system of workstations, either $\mathrm{p} 4$ or pvm are needed. Both are available from netlib (netlibeornl.gov); we have included a recent version of $\mathrm{p} 4$ in the file p4-1.2.tar. $\mathrm{z}$ in the /pub/mpi directory for convenience.

\section{Basic Routines for Point-to-Point Messages}

II this section we describe the routines that form the lowest level of the implementation. They are designed to be consistent with the upper-level routines. 


\subsection{General Communication Control}

MPI_cancel

MPI_cancel -..- ('ancel a previously initiated nonblocking send or receive

\section{Input Parameter}

$$
\text { msgid Message id returned by a call to a nonblocking send or receive }
$$

Synopsis

int MPI_cancel( msgid)

int msgid;

\section{Location}

mpi.c

MPI_infos - Determine the source process of a pending receive

Synopsis

$$
\text { int MPI_infos() }
$$

\section{Returns}

The source of the just received mesitge.

\section{Location}

$\operatorname{mpi} \cdot \mathrm{h}$

MPI_infot De.terminn the type of the last receive

Sylupsis

int MPI_infot() 


\section{Returns}

The source of the just received message.

\section{Location}

mpi.h

MPI_probe

MPI_probe -- ('heck for pending messages

\section{Input Parameters}

source the PID of the process sending the message

type: the message type

\section{Returns}

Length of pending message if available, else -1 .

\section{Synopsis}

int MPI_probe ( source, type)

int source, type;

\section{Location}

$\operatorname{mpi.c}$

MPI_stats

MPI_stats

MPI_stats - (heck the status of a nonblocking send or recejve

\section{Input Parameter}

msg_id Message id returned by a call to a nonblocking send or receive

\section{Returns}

Length of available message if it is pending, else -1 .

\section{Synopsis}

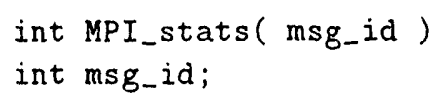




\section{Location}

$\operatorname{mpi} . \mathrm{c}$

MPI_wait

MPI_wait

MPI_wait -.- Block until a nonblocking send or receive operation has completed

\section{Input Parameter}

insg_id id returned by a nonblocking send or receive routine (of any type)

\section{Returns}

Number of bytes sent or received, or -1 on error.

\section{Synopsis}

int MPI_wait( $\mathrm{msg}_{-}$id )

int msg_id;

\section{Location}

mpi.c

\subsection{Contiguous Communications}

\subsubsection{General Routines}

MPI_crecv

MPI_crec:v

MPI_reerv Draft standard contiguous receive

\section{Input Parameters}

$\begin{array}{ll}\text { mode } & \text { one of "blocking," "nonblocking," or "synchronous" } \\ \text { buf } & \text { buffer to receive into } \\ \text { source } & \text { sending processor } \\ \text { type } & \text { message type } \\ \text { maxlen } & \text { maximum length in bytes of message }\end{array}$

\section{Returns}

If the mode is "nonblocking," returns the integer id of receive to be used in MPI_wait, ofte, or - 1 all error.

Ohlurwise, returns the actual length of the message in bytes, or -1 on error. 


\section{Synopsis}

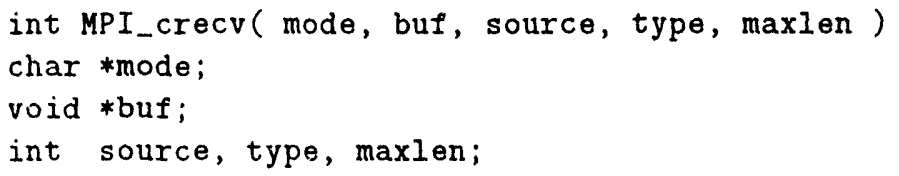

\section{Location}

mpic.c

MPI_crecvh - Friendly contiguous heterogeneous receive

\section{Input Parameters}

$\begin{array}{ll}\text { mode } & \text { one of "blocking," "nonblocking," or "synchronous" } \\ \text { buf } & \text { buffer to receive into } \\ \text { source } & \text { sending processor } \\ \text { type } & \text { message type } \\ \text { maxlen } & \text { maximum length in bytes of message } \\ \text { datatype } & \text { iype of data }\end{array}$

\section{Returns}

If the mode is "nomblocking," returns the integer id of receive to be used in MPI_wait, etc., or -1 on error.

Otherwise, returns the actual length of the message in bytes, or -1 on error.

\section{Synopsis}

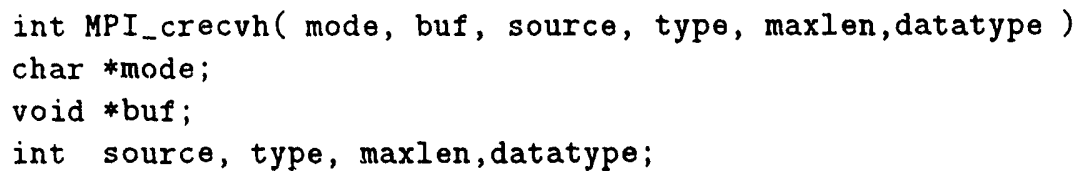

\section{Location}

mpic.c

MPI_crecvhrr - Friendly cont: guous heterogeneous receive for ready receivers

\section{Input Parameters}

mode onc of "blocking," "nonblocking," or "synchronous" 


$\begin{array}{ll}\text { buf } & \text { butfer to receive into } \\ \text { source: } & \text { sending processor } \\ \text { type } & \text { message type } \\ \text { maxlen } & \text { maximum length in bytes of message } \\ \text { datatype } & \text { type of data }\end{array}$

\section{Returns}

If the mode is "nonblocking," returns the integer id of receive to be used in MPI_wait, etc., or -1 on error.

Otherwise, returns the actual length of the message in bytes, or -1 on error.

\section{Synopsis}

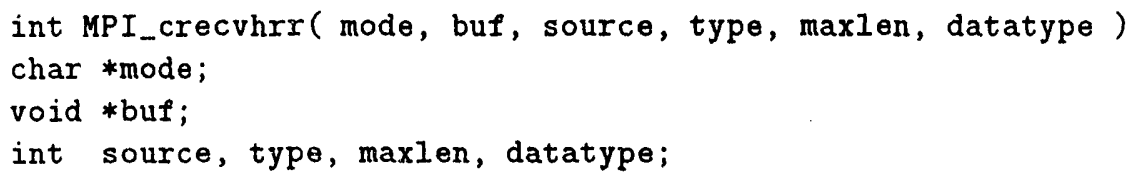

\section{Location}

mpic.c

MPI_crecvrr --- Friendly contiguous receive for ready receivers

\section{Input Parameters}

$\begin{array}{ll}\text { mode } & \text { one of "blocking," "nonblocking," or "syuchronous" } \\ \text { buf } & \text { buffer to receive into } \\ \text { source } & \text { sending processor } \\ \text { type } & \text { message type } \\ \text { maxlen } & \text { maximum length in bytes of message }\end{array}$

\section{Returns}

If the mode is "nonblocking," returns the integer id of receive to be used in MPI_wait, etc., or -1 on error.

Otherwise, returns the actual length of the message in bytes, or -1 on error.

\section{Synopsis}

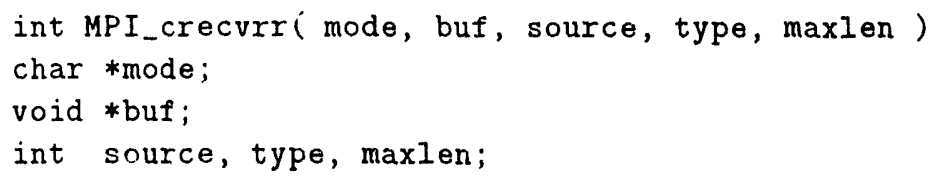




\section{Location}

mpic.c

MPI_isend

MPI_rsend

MPI_csend -- Draft standard contiguous send

\section{Input Parameters}

$\begin{array}{ll}\text { mode } & \text { one of "blocking," "nonblocking," or "synchronuns" } \\ \text { luff } & \text { buffer to send } \\ \text { dest } & \text { destination processor } \\ \text { type } & \text { message type } \\ \text { len } & \text { length in bytes of message }\end{array}$

\section{Returns}

If the mode is "nonblocking," returns the integer id of send to be used in MPI_wait, etc., or -1 on error.

Otherwise, returns the actual length of the message in bytes, or -1 on error.

Synopsis

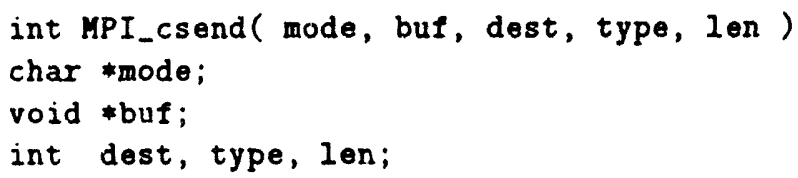

\section{Location}

mpic.c

MPI_esendh

MPI_csendh

MPI_csendh . Friendly contiguous heterogeneous send

\section{Input Parameters}

nowde?

binf

one of "blocking," "nonblocking," or "synchronous"

hinf

butfer to send

type

destination processor

leil

message type

datatype

l.ngth in bytes of message

type of datit 


\section{Returns}

If the modr is nonblocking," returns the integer id of send to be used in MPI_wait, otc.. or -1 on errur.

()therwise, returis the actual length of the message in bytes, or -1 on error.

\section{Synopsis}

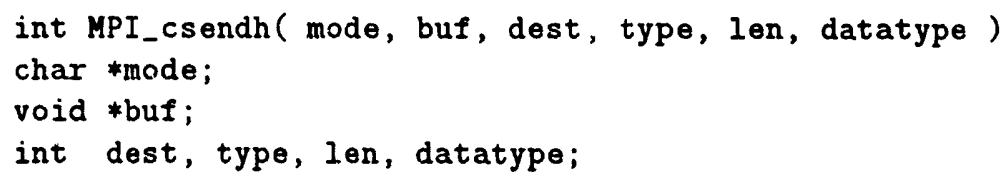

\section{Location}

mpic.c

MPI_csendhrr - Friendly contiguous h terogeneous send for ready receivers

\section{Input Parameters}

$\begin{array}{ll}\text { mode } & \text { one of "blocking," "nonblocking," or "synchronous } \\ \text { buf } & \text { buffer to send } \\ \text { dest } & \text { destination processor } \\ \text { type } & \text { message type } \\ \text { len } & \text { length in bytes of message } \\ \text { datatype } & \text { type of data }\end{array}$

\section{Returns}

If the mode is "nonblocking," returns the integer id of send to be used in MPI_wait, itc., or -1 on error.

()therwise. returus the actual length of the message in bytes, or -1 on crror.

\section{Synopsis}

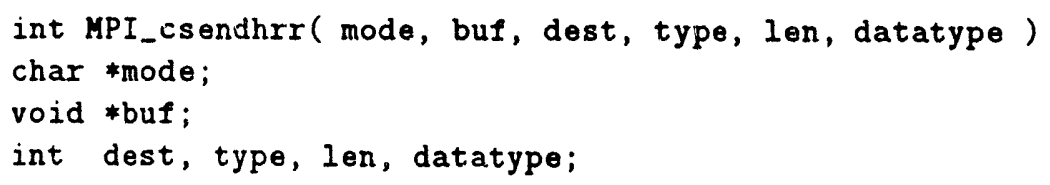

\section{Location}

$\operatorname{mpjc.c}$ 
MPI_csendrr -.. Friendly contiguous send for ready receivers

\section{Input Paraneters}

$\begin{array}{ll}\text { mode? } & \text { one of "blocking," "nonblocking," or "synchronous" } \\ \text { buf } & \text { buffer to send } \\ \text { dest } & \text { destination processor } \\ \text { type } & \text { message type } \\ \text { len } & \text { length in bytes of message }\end{array}$

\section{Returns}

If the mode is "nonbloc: ng," returns the integer id of send to be used in MPI_wait. otc., or -1 on error.

Otherwise, returns the actual length of the message in bytes, or -1 on error.

\section{Synopsis}

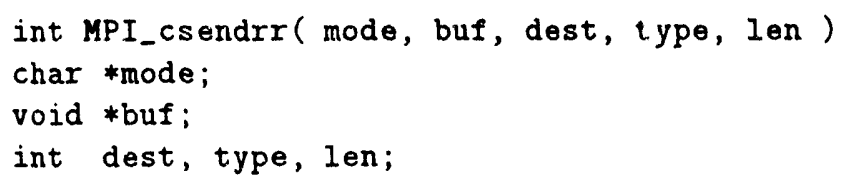

\section{Location}

mpic.c

\subsubsection{Blocking Routines}

MPI_cbrecv -- Blocking contiguous receive

\section{Input Parameters}

$\begin{array}{ll}\text { buf } & \text { buffer to receive into } \\ \text { source } & \text { sending processor } \\ \text { type } & \text { message type } \\ \text { maxlen } & \text { maximum length in bytes of inessage }\end{array}$

\section{Returns}

Number of bytes actually received. 
Synopsis

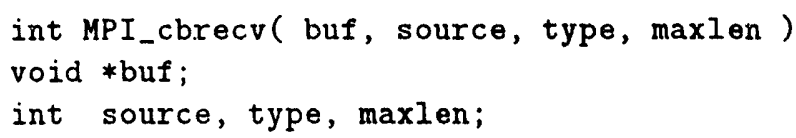

Location

mpic.c

MPI_cbrecvh MPI_cbrecevh

MPI_cbrecvh - Blocking contiguous heterogeneous receive

Input Parameters

$\begin{array}{ll}\text { buf } & \text { buffer to receive into } \\ \text { source } & \text { sending processor } \\ \text { type } & \text { message type } \\ \text { maxlen } & \text { maximum length in bytes of message } \\ \text { datatype } & \text { type of data }\end{array}$

Returns

Number of bytes actually received.

\section{Synopsis}

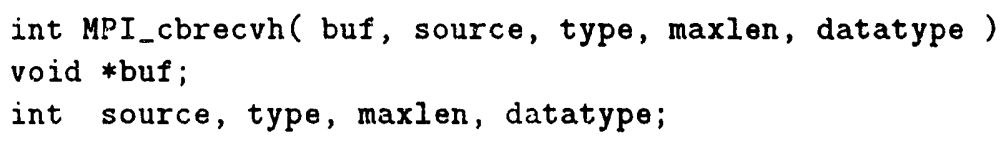

\section{Location}

mpic.c

Mli_chreevhrr

MPI_chrecturr -..- Blocking contiguous heterogeneous receive for ready receivers

\section{Input Parameters}

$\begin{array}{ll}\text { buf } & \text { buffer to receive into } \\ \text { somee } & \text { sending processor } \\ \text { type } & \text { message type } \\ \text { maxlen } & \text { maximun length in bytes of message } \\ \text { datatype } & \text { type of data }\end{array}$




\section{Returns}

Number of hytes actually received.

\section{Synopsis}

int MPI_cbrecvhrr (buf, source, type, maxlen, datatype)

void *buf;

int source, type, maxlen, datatype;

\section{Location}

mpic.c

MPI_cbrecvrr - Blocking contiguous receive for ready receivers

\section{Input Paraneters}

$\begin{array}{ll}\text { buf } & \text { buffer to receive into } \\ \text { source } & \text { sending processor } \\ \text { type } & \text { message type } \\ \text { maxlen } & \text { maximum length in } 1 \text {, tes of message }\end{array}$

\section{Returns}

Number of hytes actually received.

\section{Synopsis}

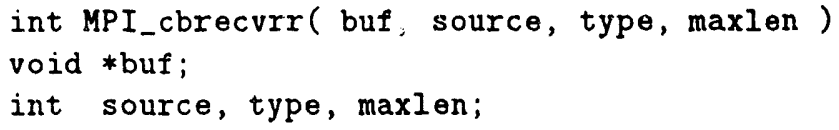

Location

mpic.c

MPI_cbsend -..- Blocking contiguous send

\section{Input Parameters}

$\begin{array}{ll}\text { buf } & \text { buffer to send } \\ \text { dest } & \text { destination }\end{array}$


$\begin{array}{ll}\text { type } & \text { message type } \\ \text { lon } & \text { length in bytes of message }\end{array}$

Returns

Number of bytes sent, or -1 for an error.

\section{Synopsis}

int MPI_cbsend ( buf, dest, type, len )

void *buf;

int dest, type, len;

\section{Location}

mpic.c

MPI_cbsendh -- Blocking contiguous heterogeneous send

\section{Input Parameters}

$\begin{array}{ll}\text { buf } & \text { buffer to send } \\ \text { dest } & \text { destination } \\ \text { type } & \text { message type } \\ \text { len } & \text { length in bytes of message } \\ \text { datatype } & \text { type of data }\end{array}$

\section{Returns}

Number of bytes sent, or -1 for an error.

\section{Synopsis}

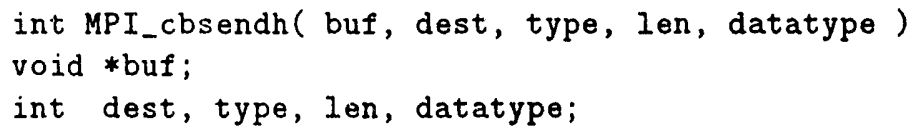

\section{Location}

mpic.c 


\section{Input Parameters}

$\begin{array}{ll}\text { buf } & \text { buffer to send } \\ \text { dest } & \text { destination } \\ \text { type } & \text { message type } \\ \text { len } & \text { length in bytes of message } \\ \text { datatype } & \text { type of data }\end{array}$

Returns

Number of hytes sent, or -1 for an error.

\section{Synopsis}

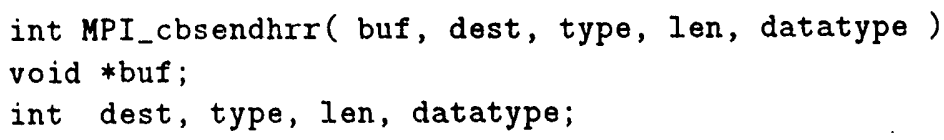

Location

mpic.c

MPI_cbsendrr --.. Blocking contiguous send for ready receivers

\section{Input Parameters}

$\begin{array}{ll}\text { buf } & \text { buffer to send } \\ \text { dest } & \text { destination } \\ \text { type } & \text { nussage type } \\ \text { len } & \text { length in bytes of message }\end{array}$

\section{Returns}

Number of bytes sent, or -1 for an error.

\section{Synopsis}

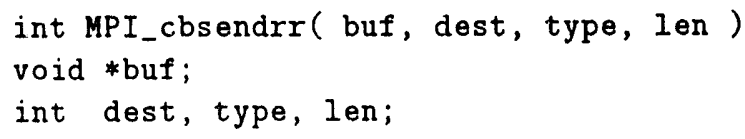

\section{Location}

mpic.c 


\subsubsection{Nonblocking Routines}

MPI_cnrecv

MPI_cureev -- Nonblocking contiguous receive

\section{Input Parameters}

$\begin{array}{ll}\text { buf } & \text { buffer to receive into } \\ \text { source } & \text { sending processor } \\ \text { type } & \text { message type } \\ \text { maxlen } & \text { maximum length in bytes of message }\end{array}$

\section{Returns}

Integer id of receive to be used in MPI_wait, etc., or -1 on error.

Synopsis

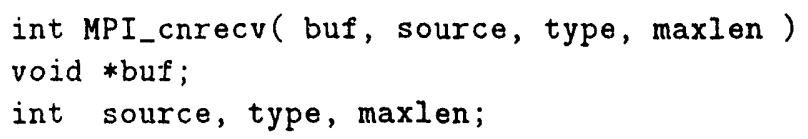

Location

mpic.c

MPI_cnrecovh -- Noriblocking contiguous heterogeneous receive

\section{Input Parameters}

$\begin{array}{ll}\text { buf } & \text { buffer to receive into } \\ \text { source } & \text { sending processor } \\ \text { type } & \text { message type } \\ \text { maxlen } & \text { maximum length in bytes of message } \\ \text { datatype } & \text { type of data }\end{array}$

\section{Returns}

Integer id of receive to be used in MPI_wait, etc., or -1 on error.

\section{Synopsis}

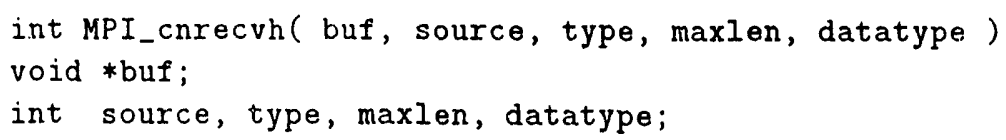




\section{Location}

mpic.c

MPI_cnrecvhrr

MPI_cnrec:vhre

MPI_cnrecvhrr -- Nonblocking contiguous heterogeneous receive for ready receivers

\section{Input Parameters}

$\begin{array}{ll}\text { buf } & \text { buffer to receive into } \\ \text { source } & \text { sending processor } \\ \text { type } & \text { message type } \\ \text { maxlen } & \text { maximum length in bytes of message } \\ \text { datatype } & \text { type of data }\end{array}$

\section{Returns}

Integer id of receive to be used in MPI_wait, etc., or -1 on error.

\section{Synopsis}

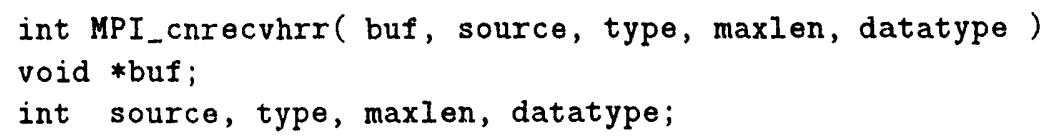

\section{Location}

mpic.c

MPI_cmrecvrr - Nonblocking contiguous receive for ready receivers

\section{Input Parameters}

$\begin{array}{ll}\text { buf } & \text { buffer to receive into } \\ \text { source } & \text { sending processor } \\ \text { type } & \text { message type } \\ \text { maxlen } & \text { maximum length in bytes of message }\end{array}$

\section{Returns}

Integer id of receive to be used in MPI_wait, etc., or -1 on error. 
Synopsis

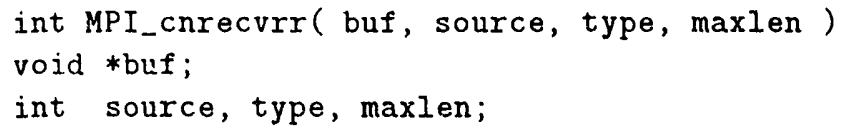

\section{Location}

mpic.c

MPI_cinse:nd

MPI_cnsend - Nonblocking contiguous send

\section{Input Parameters}

$\begin{array}{ll}\text { buf } & \text { buffer to send } \\ \text { dest } & \text { destination } \\ \text { type: } & \text { message type } \\ \text { len } & \text { length in bytes of message }\end{array}$

\section{Returns}

Integier id of send to be used in MPI_wait, etc., or -1 on error.

\section{Synopsis}

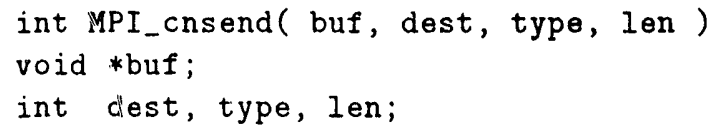

\section{Location}

mpic.c

MPI_cnsendh

MPI_(nsemdh -- Nomblocking contignous heterogeneous send

\section{Input Parameters}

$\begin{array}{ll}\text { buf } & \text { linfer to send } \\ \text { dest } & \text { clestination } \\ \text { type } & \text { messinge type } \\ \text { len } & \text { length in bytes of message } \\ \text { datatype } & \text { type of data }\end{array}$




\section{Returns}

Integer id of somd to be used in MPI_wait, etc., or -1 on error.

\section{Synopsis}

int MPI_cnsendh ( buf, dest, type, len, datatype)

void *buf;

int dest, type, len, datatype;

Locauion

$\operatorname{mpic}, \mathrm{c}$

MPI_cusendhrr

MPI_cnsendhrr

MPI_(nsendhrr --.. Nonblocking contiguous heterogeneous send for ready receivers

Input Parameters

$\begin{array}{ll}\text { buf } & \text { buffer to send } \\ \text { dest } & \text { destination } \\ \text { type } & \text { message type } \\ \text { len } & \text { length in bytes of message } \\ \text { datatype } & \text { type of data }\end{array}$

Returns

Integer id of send to be used in MPI_wait, et.c., or -1 on error.

\section{Synopsis}

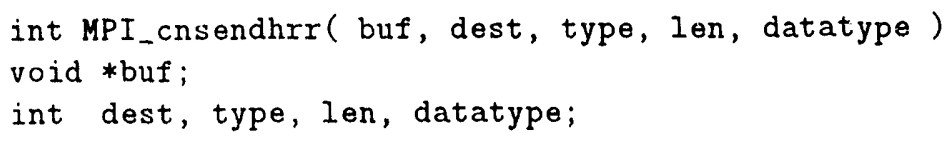

\section{Location}

mpic.c

MPI_cnsendrr - Nonblocking contiguous send for ready receivers

\section{Input Parameters}

buf buffer to receive into 


$\begin{array}{ll}\text { somree } & \text { sending processor } \\ \text { type } & \text { messiage type } \\ \text { maxlen } & \text { maximum length in hytes of message }\end{array}$

\section{Returns}

Integer id of send to be used in MPl_wait, etc., or - 1 on error.

\section{Synopsis}

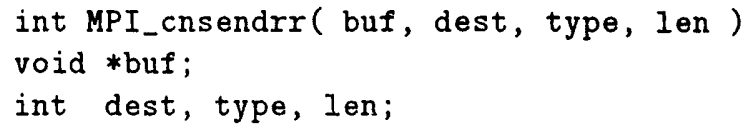

\section{Location}

mpic.c

\subsection{Constant Stride Communications}

\subsubsection{General Routines}

MPI_siecev

MPI_srecv -- Draft standard receive into buffer with constant stride

\section{Input Parameters}

\begin{tabular}{|c|c|}
\hline mode: & one of "blocking," "nonblocking," or "synchronous" \\
\hline buf & huffer to receive into \\
\hline soluese & sending processor \\
\hline type: & mossiage type \\
\hline lonlilk & size in bytes of each data block \\
\hline striclu: & IIImmber of bytes between the start of each data hlock \\
\hline llks & maximum number of data blocks \\
\hline
\end{tabular}

Returns

If the mode is "nonhlocking," returns the integer id of receive to be used in MII_wait, "t.c., or -1 on error.

()therwise, rourns the actual length of the message in bytes, or - 1 on error.

Synopsis

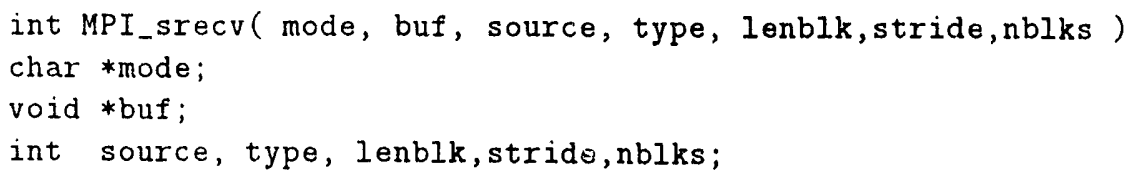




\section{Location}

mpis.c

MPI_srecvh

MPI_sreecvh

MPI_srecvh - - Friendly heterogeneous receive into buffer with constant stride

\section{Input Parameters}

$\begin{array}{ll}\text { mode } & \text { one of "blocking," "nonblocking," or "synchronous" } \\ \text { buf } & \text { buffer to receive into } \\ \text { source } & \text { sending processor } \\ \text { type } & \text { message type } \\ \text { lenblk } & \text { size in bytes of each data block } \\ \text { stride } & \text { number of bytes between the start of each data block } \\ \text { nblks } & \text { maximum number of data blocks } \\ \text { datatype } & \text { type of data }\end{array}$

\section{Returns}

If the mode is "nonblocking," returns the integer id of receive to be used in MPl_wait, et.., or -1 on error.

Otherwise, returns the actual length of the message in bytes, or -1 on error.

\section{Synopsis}

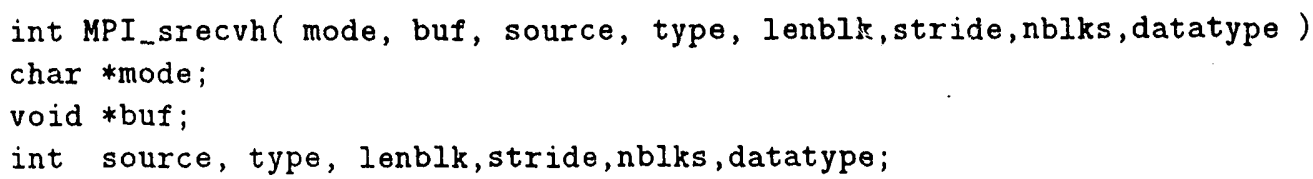

\section{Location}

mpis.c

MPI_srecvhrr - Friendly heterogeneous receive into a buffer with constant stride for ready receivers

\section{Input Parameters}

$\begin{array}{ll}\text { mode } & \text { one of "blocking," "nonblocking," or "synchronous" } \\ \text { buf } & \text { buffer to receive into } \\ \text { source } & \text { sending processor } \\ \text { type } & \text { message type } \\ \text { lenblk } & \text { size in bytes of each data hlock } \\ \text { stricke } & \text { number of bytes between the start of each data block }\end{array}$


nblks maximum number of data blocks

datatype type of data

\section{Returns}

If the mode is "nonblocking," returns the integer id of receive to be used in MP!_wait, "tu., or -1 on error.

Otherwise, returns the actual length of the message in bytes, or -1 on error.

\section{Synopsis}

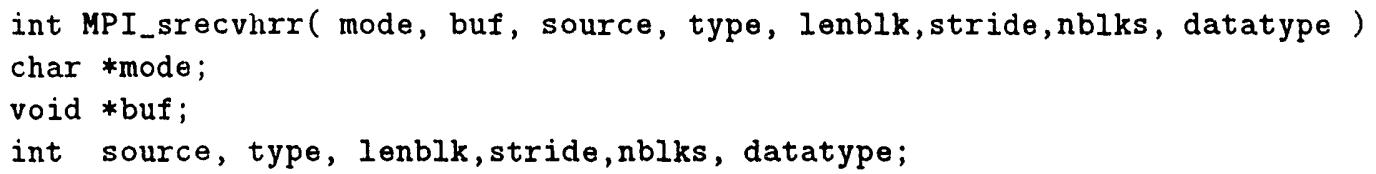

\section{Location}

mpis. c

MPI_srec:vrr ….. Friendly receive into buffer with constant stride for ready receivers

\section{Input Parameters}

\begin{tabular}{|c|c|}
\hline mode & one of "blocking," "nonblocking," or "synchronous" \\
\hline buf & buffer to receive into \\
\hline sollece & sending processor \\
\hline type: & inessage type \\
\hline lenblk & size in bytes of rach data block \\
\hline strirle & number of bytes lutween the start of each data block \\
\hline nthlks & maximum number of lata blocks \\
\hline
\end{tabular}

\section{Returns}

If the mode is "monblocking," returns the integer id of receive to be used in MPI_wait, etc., or -1 on error.

()therwise, returns the actual length of the message in bytes, or -1 on error.

\section{Synopsis}

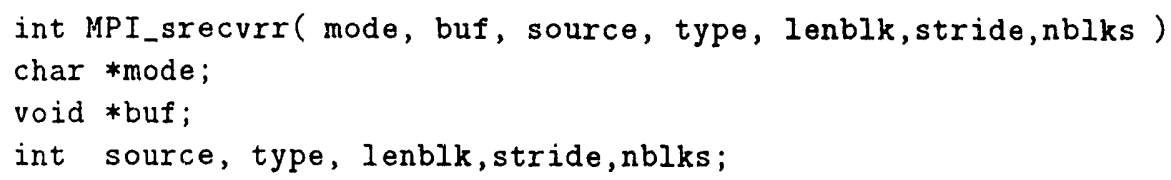

\section{Location}

mpis. c 
MPI_ssend - Draft standard send with constant stride

\section{Input Parameters}

$\begin{array}{ll}\text { modes } & \text { one of "blocking." "nomblocking." or "synchronous" } \\ \text { buf } & \text { huffer to send } \\ \text { dest } & \text { destinat: in processor } \\ \text { type } & \text { message type } \\ \text { lenblk } & \text { size in bytes of each data olock } \\ \text { stride } & \text { number of bytes between the start of each data blork } \\ \text { nblks } & \text { number of data blocks }\end{array}$

\section{Returns}

If the node is "nonblocking." returns the integer id of send to he used in MPI_wait. etc., or -1 on error.

Otherwise, returns the actual length of the message in bytes, or - 1 on error.

\section{Synopsis}

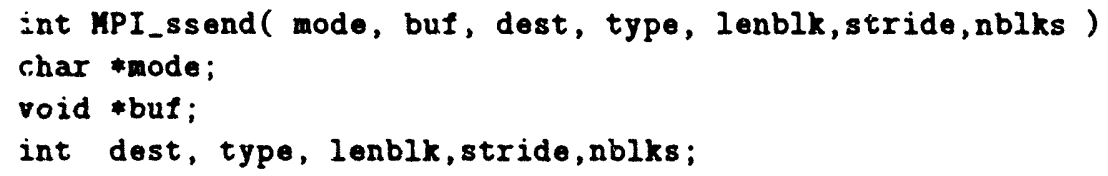

\section{Location}

apis.c

MPI_sseudh - Fripndly heterogeneous send with constant stride

\section{Input Parameters}

$\begin{array}{ll}\text { mode" } & \text { one of "blocking." "nonblocking." or "synchronous" } \\ \text { buf } & \text { lutfer to send } \\ \text { dest } & \text { destination processor } \\ \text { type } & \text { nuessage type } \\ \text { leublk } & \text { size in bytes of each data block } \\ \text { stride } & \text { number of bytes between the start of each data bluck } \\ \text { ublks } & \text { number of data blocks } \\ \text { datatype. } & \text { Iy) of data }\end{array}$




\section{Returns}

If the mode is "nonblocking," returns the integer id of send to he used in MPI_wait, itc., or -1 on error.

Otherwise, returns the actual length of the message in bytes, or -1 on error.

\section{Synopsis}

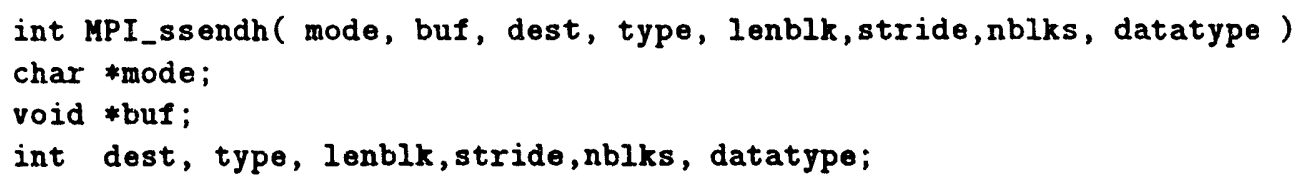

\section{Location}

mpis.c

MPI_ssendhrr - Friendly heterogeneous send with constant stride for ready receivers

\section{Input Parameters}

$\begin{array}{ll}\text { mode } & \text { one of "blocking," "nonblocking," or "synchronous" } \\ \text { buf } & \text { buffer to send } \\ \text { dest } & \text { destination processor } \\ \text { type: } & \text { message type } \\ \text { lenblk } & \text { size in bytes of each data block } \\ \text { stride: } & \text { number of bytes between the start of each data block } \\ \text { nblks } & \text { number of dat a blocks } \\ \text { datatype } & \text { type of data }\end{array}$

\section{Returns}

If the mode is "nonblocking," returns the integer id of send to be used in MPI_wait, et.e., or -1 on error.

()therwise, ruturns the actual length of the message in bytes, or -1 on error.

\section{Synopsis}

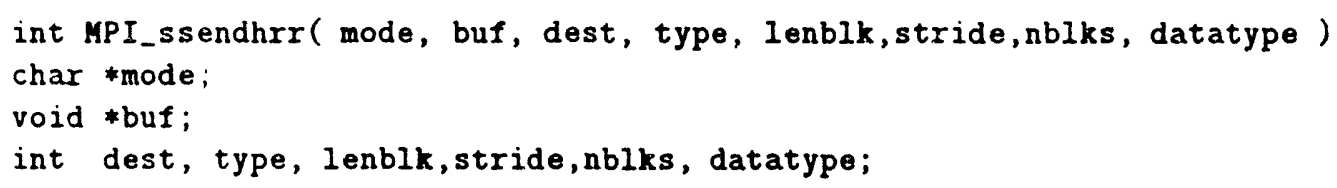

\section{Location}

mpis.c 
MPI_ssendrr ...- Friendly send with constant stride for ready receivers

\section{Input Parameters}

$\begin{array}{ll}\text { mode: } & \text { one of "blocking," "nonblocking," or "synchronous" } \\ \text { buf } & \text { buffer to send } \\ \text { dest } & \text { destination processor } \\ \text { type } & \text { message type } \\ \text { lenblk } & \text { size in bytes of each data block } \\ \text { stride: } & \text { number of bytes between the start of each data block } \\ \text { nblks } & \text { number of data blocks }\end{array}$

\section{Returns}

If the mode is "nonblocking," returns the integer id of send to be used in MPI_wait. etc., or -1 on error.

Otherwise, returns the actual length of the message in bytes, or -1 on error.

\section{Synopsis}

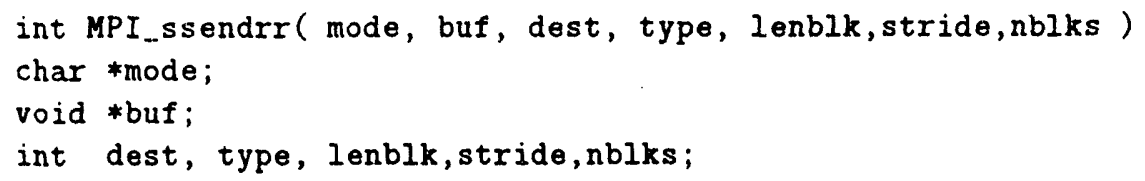

\section{Location}

mpis.c

\subsubsection{Blocking Runtines}

MPI_sbrecv -- Blocking receive into buffer with constant stride

\section{Input. Parameters}

$\begin{array}{ll}\text { buf } & \text { buffer to receive into } \\ \text { sumree } & \text { sending processor } \\ \text { types } & \text { message type } \\ \text { lenblk } & \text { size in bytes of each data block } \\ \text { stride: } & \text { mumber of bytes between the start of each data blork } \\ \text { nblks } & \text { maximum number of data blocks }\end{array}$




\section{Returns}

Number of hylus actually received.

\section{Synopsis}

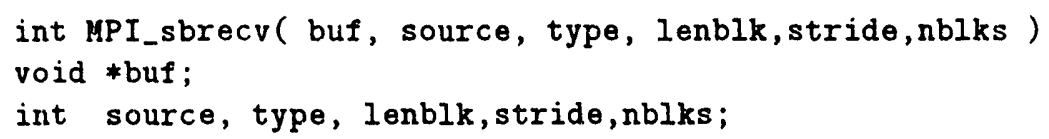

\section{Location}

mpis.c

MPI_shrecvh --.- Blocking heterogeneous receive into buffer with constant stride

\section{Input Parameters}

$\begin{array}{ll}\text { buf } & \text { buffer to receive into } \\ \text { source } & \text { sending processo: } \\ \text { type } & \text { message type } \\ \text { lenblk } & \text { size in bytes of each data block } \\ \text { stride } & \text { number of bytes between the start of each data block } \\ \text { nblks } & \text { maximum number of data blocks } \\ \text { datatype } & \text { type of data }\end{array}$

\section{Returns}

Number of bytes actually receiverd.

\section{Synopsis}

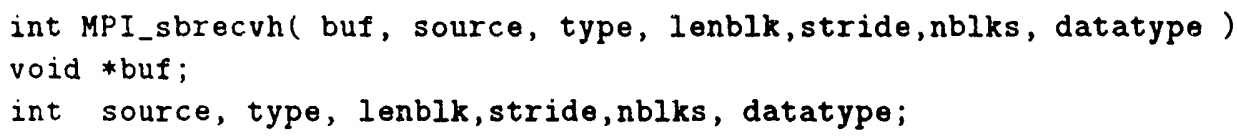

\section{Location}

mpis.c

MPI_shrecvhrr - Blocking heterogeneous receive into buffer with constant stride for ready receivers 


\section{Input Parameters}

$\begin{array}{ll}\text { buf } & \text { buffer to receive into } \\ \text { source } & \text { sending processor } \\ \text { type } & \text { message type } \\ \text { lenblk } & \text { size in bytes of each data block } \\ \text { stride } & \text { number of bytes between the start of each data blork } \\ \text { nblks } & \text { maximum number of data blocks } \\ \text { datatype } & \text { type of data }\end{array}$

\section{Returns}

Number of hytes actually received.

\section{Synopsis}

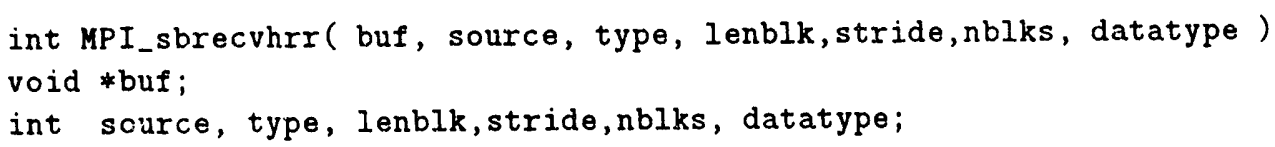

\section{Location}

mpis.c

MPI_sbrecvrr - Blocking receive into buffer with constant stride for ready receivers

\section{Input Parameters}

$\begin{array}{ll}\text { buf } & \text { buffer to receive into } \\ \text { source } & \text { sending processor } \\ \text { type } & \text { message type } \\ \text { lenblk } & \text { size in bytes of each data block } \\ \text { stride } & \text { number of bytes between the start of each data block } \\ \text { nblks } & \text { maximum number of data blocks }\end{array}$

\section{Returns}

Number of hytes actually received.

\section{Synopsis}

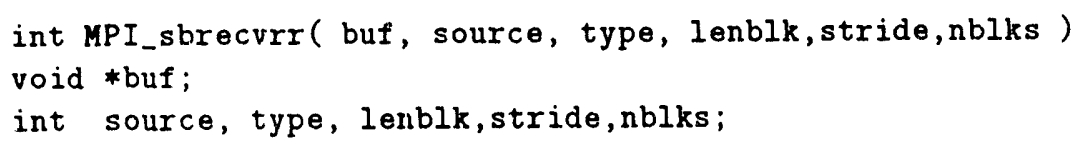

\section{Location}

mpis. c 
MPI_sbsend -.. Blocking send with constant stride

\section{Input Parameters}

$\begin{array}{ll}\text { buf } & \text { buffer to send } \\ \text { dest } & \text { destination } \\ \text { type } & \text { message type } \\ \text { lenblk } & \text { size in bytes of each data block } \\ \text { stride } & \text { number of bytes between the start of each data block } \\ \text { nblks } & \text { number of data blocks }\end{array}$

\section{Returns}

Number of bytes sent, or -1 for an error.

\section{Synopsis}

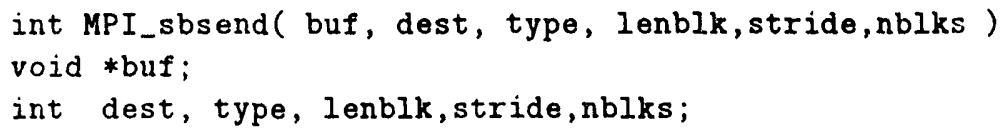

\section{Location}

mpis.c

MPI_sbsendh - Blocking heterogeneous seml with constant stride

\section{Input Parameters}

$\begin{array}{ll}\text { linf } & \text { buffer to send } \\ \text { dest } & \text { destination } \\ \text { type } & \text { message type } \\ \text { lenblk } & \text { size in bytes of each data block } \\ \text { stride } & \text { number of bytes between the start of each data block } \\ \text { nblks } & \text { number of data blocks } \\ \text { datatype: } & \text { type of data }\end{array}$

\section{Returns}

Number of hetes semt, or - l for an error. 


\section{Synopsis}

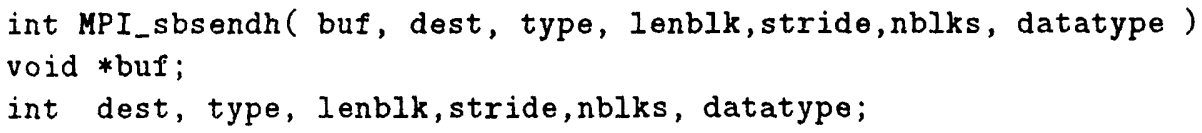

\section{Location}

mpis.c

MPI_sbsendhrr - Blocking heterogeneous send with constant stride for ready receivers

\section{Input Parameters}

$\begin{array}{ll}\text { buf } & \text { buffer to send } \\ \text { dest } & \text { destination } \\ \text { type } & \text { message type } \\ \text { lenblk } & \text { size in bytes of each data block } \\ \text { stride } & \text { number of bytes between the start of each data block } \\ \text { nblks } & \text { number of data blocks } \\ \text { datatype } & \text { type of data }\end{array}$

\section{Returns}

Number of bytes sent, or -1 for an error.

\section{Synopsis}

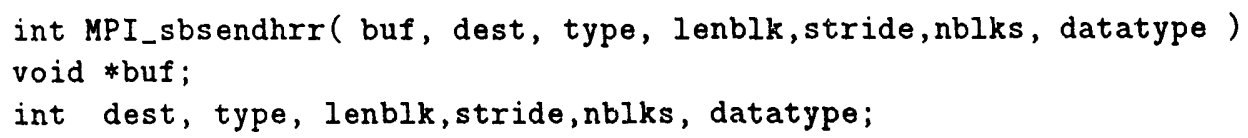

\section{Location}

mpis. c

MPI_sbsendrr - - Blocking send with constant stride for ready receivers

\section{Input Parameters}

$\begin{array}{ll}\text { buf } & \text { buffer to send } \\ \text { lesst } & \text { lestination } \\ \text { type } & \text { message type } \\ \text { lenblk } & \text { size in hytes of each data block }\end{array}$


stride number of bytes between the start of each data block nblks number of data blocks

\section{Returns}

Number of bytes sent, or -1 for an error.

Synopsis

int MPI_sbsendrr ( buf, dest, type, lenblk, stride,nblks )

void *buf;

int dest, type, lenblk, stride,nblks;

Location

mpis.c

\subsubsection{Nonblocking Rontines}

MPI_surecv -... Nonblocking receive into buffer with constant stride

\section{Input Parameters}

$\begin{array}{ll}\text { buf } & \text { buffer to receive into } \\ \text { somrce } & \text { sending processor } \\ \text { type: } & \text { message type } \\ \text { lemblk } & \text { size in bytes of cach data block } \\ \text { stride: } & \text { mumber of bylus lictwern the start of each data block } \\ \text { nblks } & \text { maximum number if data blocks }\end{array}$

\section{Returns}

Integer id of recoive to be used in MPl_wail. etce, or - l on error.

Synopsis

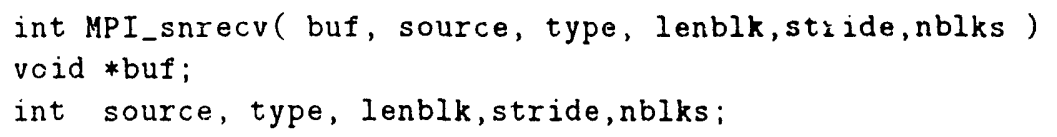

\section{Location}

mpis.c 
MPI_surecevh -... Nonblocking heterogeneous receive into buffer with constant stride

\section{Input Parameters}

\begin{tabular}{|c|c|}
\hline $\begin{array}{l}\text { buf } \\
\text { source }\end{array}$ & $\begin{array}{l}\text { Dutfer to receive into } \\
\text { semding processor }\end{array}$ \\
\hline type: & message typer \\
\hline lenblk & size in bytes of each lata block \\
\hline stride: & number of bytes between the start of each datia blerk \\
\hline nlblks & maximum number of data blocks \\
\hline datatype: & type of clata \\
\hline
\end{tabular}

\section{Returns}

Integer id of receive to be used in MPI_wait, etc., or - I on orror.

\section{Synopsis}

int MPI_snrecvh( buf, source, type, lenblk,stride,nblks, datatype)

void *buf;

int source, type, lenblk, stride,nblks, datatype;

\section{Location}

mpis.c

MPI_snrecevhrr Nonlikecking heterogeneous reccive into buffer with comstant stride for ready recrivirs

\section{Input Parameters}

\begin{tabular}{|c|c|}
\hline buf & buffer to receive into \\
\hline sonirce & sending processor \\
\hline type: & message type \\
\hline lenhlik & sizo in bytes of each data block \\
\hline stride: & mumber of bytes between the start of each data block \\
\hline nblks & maximum number of datia blocks \\
\hline datatype: & type of aliatia \\
\hline
\end{tabular}

\section{Returns}

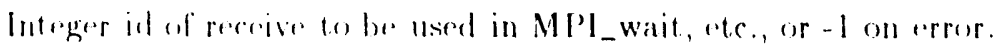




\section{Synopsis}

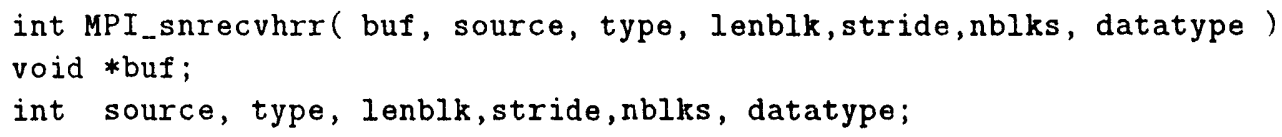

\section{Location}

mpis.c

MPI_surectvrr -... Nonblocking receive into buffer with constant stride for ready receivers

\section{Input Parameters}

$\begin{array}{ll}\text { luf } & \text { buffer to receive into } \\ \text { source } & \text { sending processor } \\ \text { type } & \text { message type } \\ \text { lenblk } & \text { size in bytes of each data block } \\ \text { stricle: } & \text { mumber of bytes between the start of each data block } \\ \text { nblks } & \text { maximum number of data blocks }\end{array}$

\section{Returns}

Integer id of receive to be used in MPI_wait, etc., or -1 on error.

\section{Synopsis}

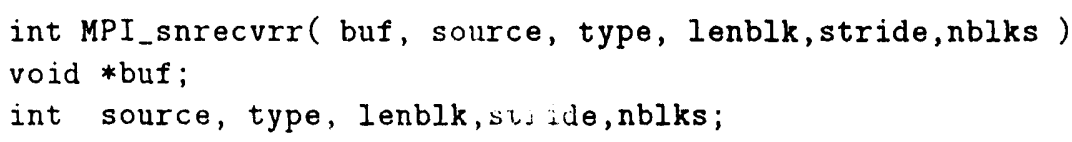

\section{Location}

mpis. c

MPI_snsemd - Nonblocking send with constant stride

\section{Input Parameters}

$\begin{array}{ll}\text { buf } & \text { bulfer to send } \\ \text { dest } & \text { lestiuntion } \\ \text { type } & \text { messige type } \\ \text { lemblk } & \text { size in bytes of each data block } \\ \text { stride: } & \text { mumber of bytes between the start of each data block }\end{array}$


nblks number of data blocks

\section{Returns}

Intrger id of send to be used in MPl_wait, ete, or -1 on error.

\section{Synopsis}

int MPI_snsend (buf, dest, type, lenblk, stride,nblks )

void *buf;

int dest, type, lenblk, stride,rblks;

\section{Location}

mpis.c

MPI_susendh

MPI_snsendh

MPI_snsendh -- Nonblocking heterogeneous send with comstant stride

\section{Input Parameters}

$\begin{array}{ll}\text { buf } & \text { buffer to send } \\ \text { dest } & \text { destination } \\ \text { type } & \text { message type } \\ \text { lenblk } & \text { size in bytes of each data block } \\ \text { stride } & \text { number of bytes between the start of each data block } \\ \text { nblks } & \text { number of data blocks } \\ \text { datatype. } & \text { type of data }\end{array}$

Returns

Integer id of send to be used in MPI_wait, etc., or -1 on error.

\section{Synopsis}

int MPI_snsendh ( buf, dest, type, lenblk, stride,nblks, datatype ) void *buf;

int dest, type, lenblk, stride,nblks, datatype;

\section{Location}

mpis.c

MPI_snsendhrr - Nonhlocking heterogeneous send with constant stride for reaty receivers 


\section{Input Parameters}

$\begin{array}{ll}\text { buf } & \text { buffer to send } \\ \text { dest } & \text { destination } \\ \text { type: } & \text { mesigat type } \\ \text { lenblk } & \text { size in bytes of each data block } \\ \text { stride } & \text { number of bytes between the start of each data block } \\ \text { nblks } & \text { number of data blocks } \\ \text { latatype } & \text { type of data }\end{array}$

\section{Returns}

Integer id of send to be used in MPI_wait, etc., or -1 on error.

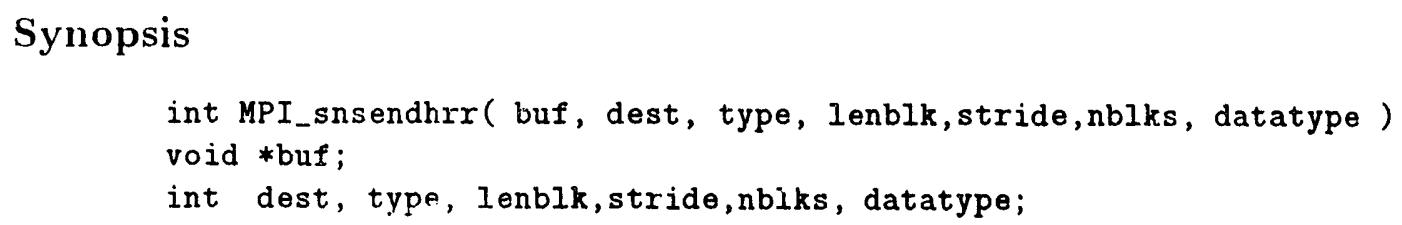

\section{Location}

mpis.c

MPI_snsendrr --.- Noublocking send with constant stride for ready receivers

\section{Input Parameters}

$\begin{array}{ll}\text { buf } & \text { buffer to receive into } \\ \text { source } & \text { semding processor } \\ \text { type } & \text { messige type } \\ \text { lenblk } & \text { size in bytes of each data block } \\ \text { strides } & \text { mumber of bytes between the start of each data block } \\ \text { nblks } & \text { number of data biocks }\end{array}$

\section{Returns}

Integer id of send to be used in MPl_wait, etc., or - 1 on error.

\section{Synopsis}

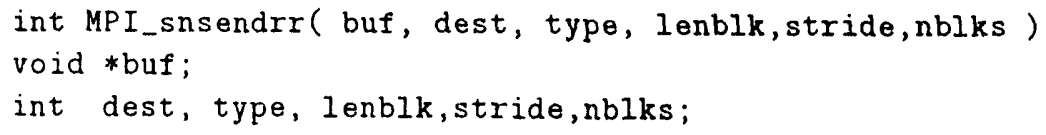

Location

mpis.c 


\subsection{General Scatter/Gather}

These routines USe structures named MPI_DATAVEC and MPI_HDATAVEC (for heterogemeoms commmmi(ation). The definitions of these are in mpi.h. MPIDATAVEC is the samb as struct iovec; this is a structure that contains a pointer to data and the size of that data in hytes. MPI.HDATAVEC alls : value that is the type of the data, in MPI format.

\subsubsection{General Routines}

MPI_greev -... Iraft standard receive into buffer with arbitrary scatter

\section{Input Parameters}

mode one of "blocking," "noublocking," or "synchrommus"

source. sending processor

type message type.

desce description of data to be scattered, in MPI_LATAVE(: format

bont numblier of blocks

\section{Returns}

If the mode is "nomblocking," returns the integer id of receive to be used in MPl_wait, ntc., or -1 on error.

Otherwise, returns the actual length of the message in bytes, or -1 on error.

\section{Synopsis}

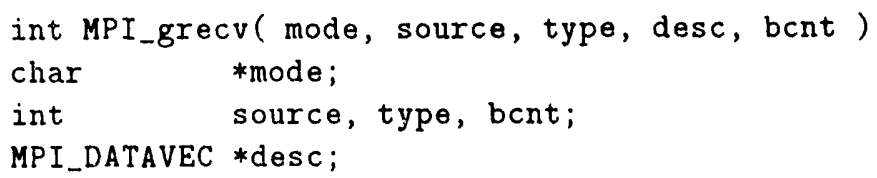

\section{Location}

mpig.c

MPI_grecevh -..- Friendly heterogeneous receive into buffer with arbitrary scatter

\section{Input Parameters}

incide:

somres:

type

disce (une of "hlocking," "nonblocking," or "synchromuns"

sculing processor

message type

description of data to be scattered, in MPI_ls'TAVEc: format. 
bent number of blocks

\section{Returns}

If the mode is "momblocking," returus the integer id of receive to be used in MPl_wait, "t."., (il) - 1 on croror.

()therwise, relurus the actual length of the message in bytes, or -1 on error.

\section{Synopsis}

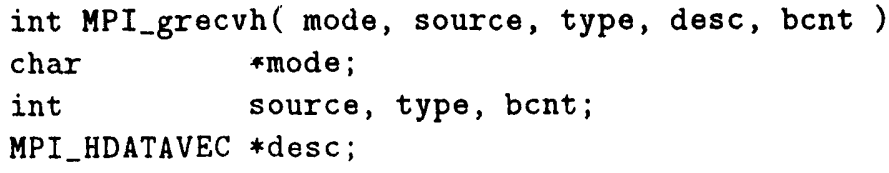

\section{Location}

$\operatorname{mpig} \cdot \mathrm{c}$

MPI_grecovhrr -... Friendly heterogeneous receive into a buffer with arbitrary scatter for ready receivers

\section{Input Parameters}

mode: One of "blocking," "nonblocking," or "yn tronous"

sources set ing processor

type: Inessage type

desc: description of tata to be scattered, in MPI_DA'lAVEc; format

bent mumber of blocks

\section{Returns}

If the mode is "unuldocking," returns the integer id of receive to be used in MPI_wait, etc., (1) -1 on crror.

()therwise, roturns the actual length of the message in bytes, or -1 on error

Synopsis

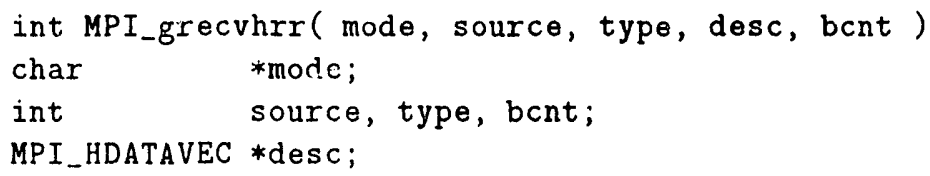

Location

mpig. c 


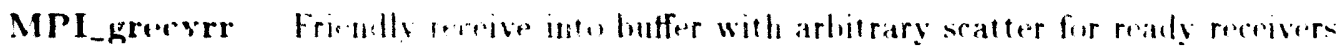

\section{Input Parameters}

inudi.

"nin of "blocking." "nomblocking." or "synchrumuss"

semere.

sinding processur

type. minciage types

disise

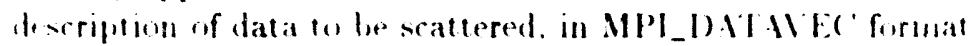

linit mumbire if locks

\section{Returus}

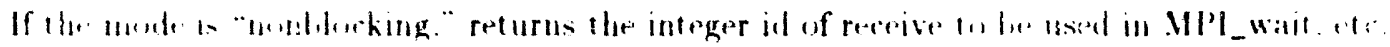
$\operatorname{ar}-1$ ind ertit

():herwion. return- the artual length of the message in bytes, or - 1 chl error

\section{Synopis}

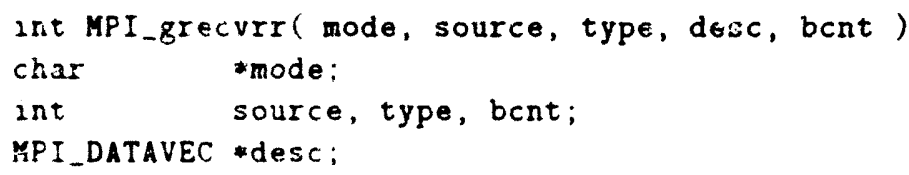

\section{Loration}

$\operatorname{mp} 28 \cdot c$

MPI_haend braft tamblat seld with arbitrary gather

\section{linput Parameters}

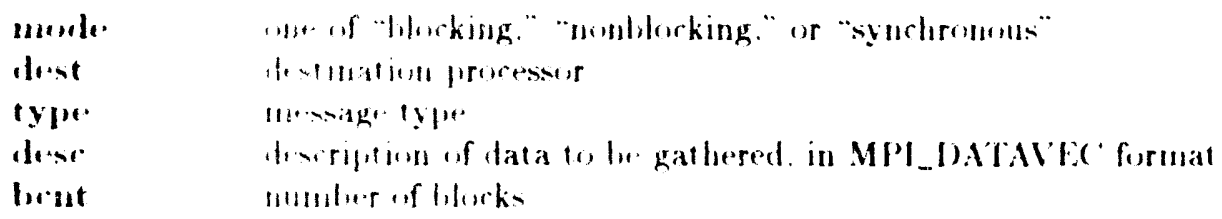

\section{Returns}

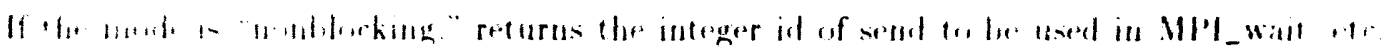

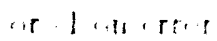

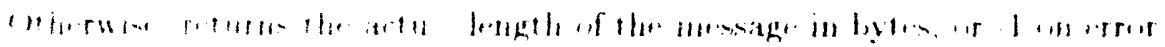


Synopsis

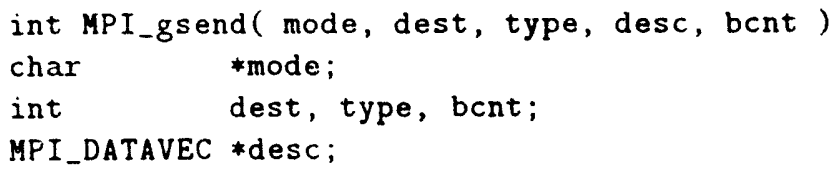

\section{Location}

$\operatorname{mpig} \cdot \mathrm{c}$

MPI_gsendh - - I iendly leeterogeneous send with arbitrary gather

\section{Input Parameters}

mescle.

oute "f "hlocking," "nonblocking," or "synchronous"

lesit destination processor

type. milessige type

desc description of data to be gathered, in MPI_DATAVE( format

lirnt numulier of blocks

\section{Returns}

If the mudr. is "monblocking." returns the integer id of send to be used in MPI_wait, ".tc.. ur -1 an erpor.

()throwse. returns the actual length of the message in bytes. or - 1 on error.

\section{Synopsis}

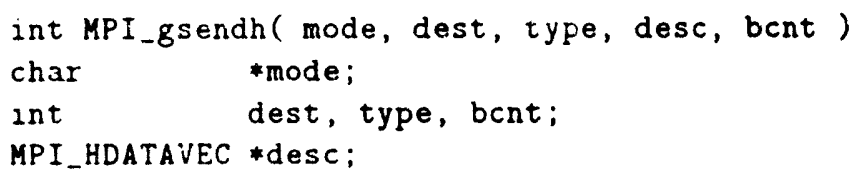

Location

Inpig.c

MPI_minendhrr

MPI_gsendhry

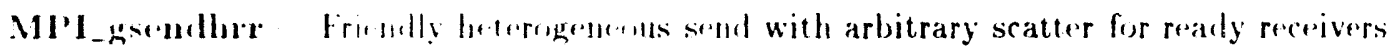

\section{Input Parameters}

morde.

"HI. of "hlocking." "nonllreking," or "synchronous"

dest

destination processor 
$\begin{array}{ll}\text { type } & \text { message type } \\ \text { dese: } & \text { lescription of data to be gathered. in MPI_IATAVEC: format. } \\ \text { bent } & \text { number of blocks }\end{array}$

\section{Returns}

If the mode is "nomblocking," returns the integer id of send to he used in Mll_wait. "ti.. or -1 on errur.

Otherwise, returns the actual length of the message in bytes, or - 1 on error.

\section{Synopsis}

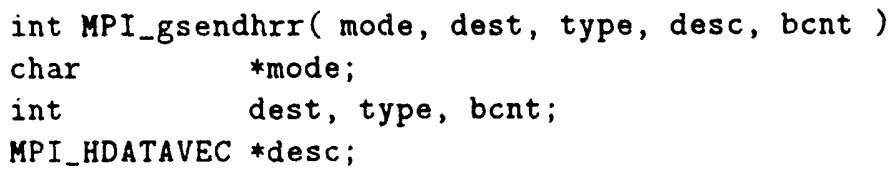

\section{Location}

mpig.c

MPI_gsendrr -... Friendly send with arbitrary gather for ready receivers

\section{Input Parameters}

$\begin{array}{ll}\text { mode. } & \text { one of "blocking," "nonblocking," or "synchronous" } \\ \text { dest } & \text { lestination processor } \\ \text { type } & \text { message type } \\ \text { desc } & \text { description of data to be gathered, in MPI_DATAVEC: format } \\ \text { bent } & \text { mumber of blocks }\end{array}$

\section{Returns}

If the mode is "nonblocking," returns the integer id of send to be used in Ml'.. wait. rte.. or - I on error.

()therwise, returns the actual length of the message in bytes, or -1 an error.

\section{Synopsis}

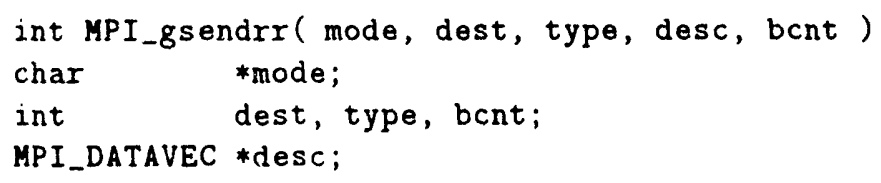

\section{Location}

mpig.c 


\subsubsection{Blocking Routiness}

MPI_gbreev

MPI_gbree:v

MPI_ghreev --- Blocking recrive into buffer with arbitrary scatter

\section{Input Parameters}

$\begin{array}{ll}\text { source } & \text { sending processor } \\ \text { type } & \text { message type } \\ \text { desc: } & \text { description of data to be scattered, in MPI_DATAVEC: format } \\ \text { bent } & \text { mumber of blocks }\end{array}$

\section{Returns}

Number of hytes actually rieived.

\section{Synopsis}

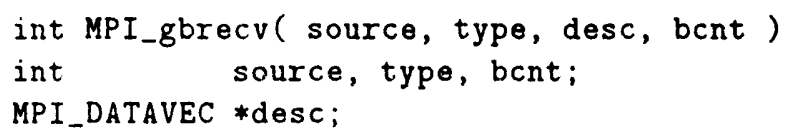

\section{Location}

mpig.c

MPI_threcvh

MPI_phreevh Blowking heterogenerus m. ive into buffer with arbitrary scatter

\section{Input Parameters}

$\begin{array}{ll}\text { somes } & \text { mending processor } \\ \text { type } & \text { messige type } \\ \text { dese } & \text { description of data to be scattered, in MPI_HDATAVEC: format } \\ \text { bent } & \text { number of blocks }\end{array}$

\section{Returns}

Nimber of ligten actually received.

Synopsis

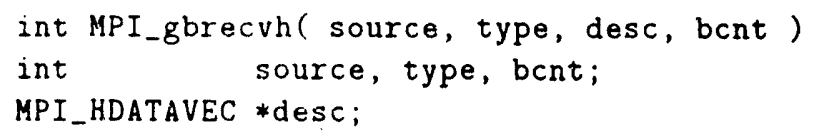




\section{Location}

mpig.c

MPI_ghrecvhrr

MPI_thrervhrr

MPI_kbrencvirr - Blocking heterogeneous receive into buffer with arbitrary scatter for realy r. ceivers

\section{Input Parameters}

$\begin{array}{ll}\text { source: } & \text { sending processor } \\ \text { type: } & \text { inesage type } \\ \text { desc } & \text { description of data to be scattered. in MPI_DATAVEC: format } \\ \text { bent } & \text { number of blocks }\end{array}$

\section{Returns}

Number of bytes actually received.

Synopsis

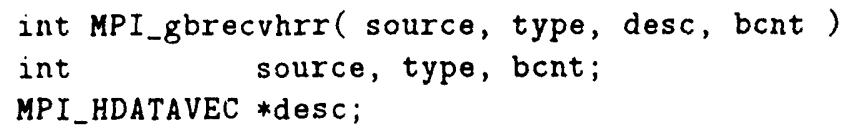

\section{Location}

$$
\text { mpig.c }
$$

MPI_gbrecvrr -.. Blocking receive into buffer with arbitrary scatter for realy receivers

\section{Input Parameters}

$\begin{array}{ll}\text { somres } & \text { sending processor } \\ \text { type: } & \text { message type } \\ \text { dese: } & \text { description of data to be scattered, in MPI_DATAVE(: format } \\ \text { bent } & \text { mumber of blocks }\end{array}$

\section{Returns}

Number of hyles artually recrived. 
Synopsis

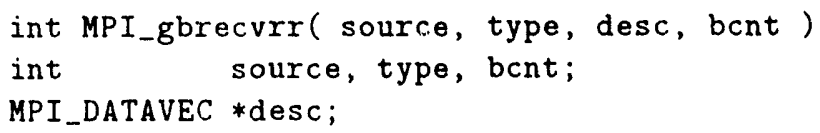

\section{Location}

$\operatorname{mpig} \cdot \mathrm{c}$

MPI_gbsend -..- Blocking send with arhitrary gather

\section{Input Parameters}

$\begin{array}{ll}\text { dest } & \text { destination } \\ \text { type } & \text { message type } \\ \text { dese: } & \text { description of data to be gathered, in MPI_DATAVEC: format } \\ \text { bent } & \text { number of blocks }\end{array}$

\section{Returns}

Number of lytes sent, or -1 for an error.

\section{Synopsis}

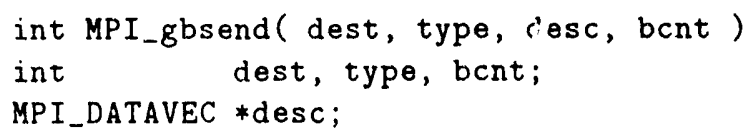

Location

mpig.c

MII_Lhoendh

MPl_mbsemelh Blorking send with arbitrary gather

\section{Input Parameters}

\begin{tabular}{|c|c|}
\hline dest & destination \\
\hline type & messige type \\
\hline lenlilk & size in bytes of each data block \\
\hline $\begin{array}{l}\text { dese: } \\
\text { bent }\end{array}$ & $\begin{array}{l}\text { Weription of data to be gathered, in MPI_DATAVE(' format } \\
\text { munulur of blocks }\end{array}$ \\
\hline
\end{tabular}




\section{Returns}

Number of hytes sent, or -1 for an error.

\section{Synopsis}

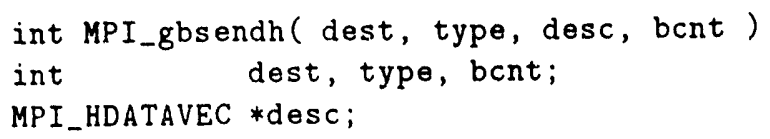

Location

mpig.c

MPI_ylusendhrr

MPI_gbsendhry

MPI_ghsendhrr -... Blocking heterogeneous send with arbitrary gather for realy recrivers

\section{Input Parameters}

$\begin{array}{ll}\text { dest } & \text { destination } \\ \text { type } & \text { message type } \\ \text { dese } & \text { description of data to be gathered, in MPI_DATAVEC : format } \\ \text { bent } & \text { number of blocks }\end{array}$

\section{Returns}

Number of bytess sent, or -1 for an error.

\section{Synopsis}

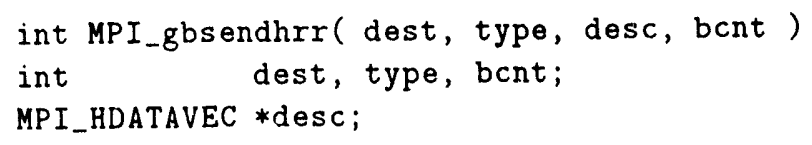

\section{Location}

$$
\text { mpig.c }
$$

MPI_nbsendrr

MPI_glsendrr … Blocking send with arbitrary gather for ready receivers

\section{Input Parameters}

$\begin{array}{ll}\text { dest } & \text { destination } \\ \text { type } & \text { message type }\end{array}$


dese Irscription of data to bo gallored, in MPI_LA'TAVLC: format

lint mumblore of blocks

Returns

Number of ligtes sent or - I for an error.

Synopsis

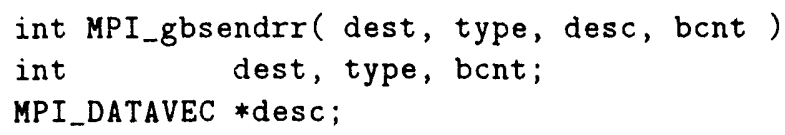

Location

mpig.c

\subsubsection{Nomblorking Routines}

MPI_gnrece Nomblraking recrive into buffer with arbitrary scatter

\section{Input Parameters}

\begin{tabular}{|c|c|}
\hline s(1)111:e? & iellding processor \\
\hline type: & mesarge type \\
\hline desc: & description of data to be scattered, in MPI_LA'TAVE(: format. \\
\hline linent & momber of blicks \\
\hline
\end{tabular}

\section{Returns}

Integer in uf receive to be used in Mll't.wat, etc., or -1 on error.

Synopsis

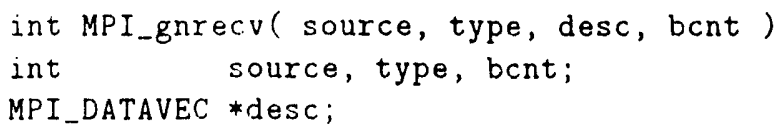

Locition

mpig. c

MPI. himerevh

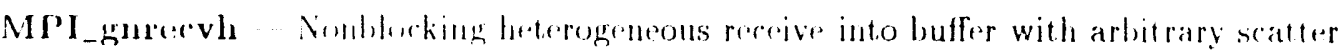


Input Parameters

$\begin{array}{ll}\text { source } & \text { sending processor } \\ \text { type } & \text { message type } \\ \text { dese } & \text { description of data to be scattered, in MPI_LATAVEC format } \\ \text { bent } & \text { mumber of blocks }\end{array}$

\section{Returns}

Integer id of receive to be used in MPI_wait, etc., or -1 on error.

\section{Synopsis}

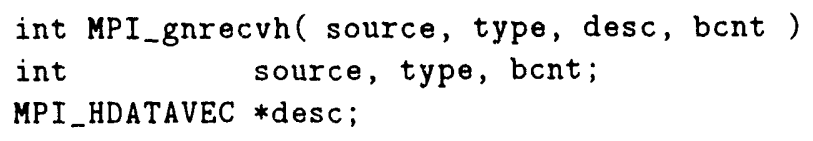

\section{Location}

mpig.c

MPI_gnrecvhrr - Nonblocking heterogeneous receive into buffer with arbitrary scatter for ready receivers

\section{Input Parameters}

$\begin{array}{ll}\text { source } & \text { sending processor } \\ \text { type } & \text { message type } \\ \text { desc } & \text { description of data to be stattered, in MPI_DATAVE: format } \\ \text { bent } & \text { number of blocks }\end{array}$

\section{Returns}

Integer id of receive to be used in $\mathrm{MPI}_{-}$wait, etc., or -1 on error.

\section{Synopsis}

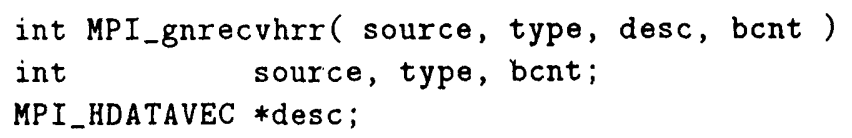

\section{Location}

mpig.c 
MPI_rnmecvrr - Nonblocking receive into buffer with arbitrary scatter for ready receivers

\section{Input Parameters}

$\begin{array}{ll}\text { source } & \text { sending processor } \\ \text { type } & \text { message type } \\ \text { desc: } & \text { description of data to be scattered, in MPI_LATAVEC format } \\ \text { bent } & \text { number of blocks }\end{array}$

\section{Returns}

Integer id of receive to be used in MPI_wait, etc., or -1 on error.

\section{Synopsis}

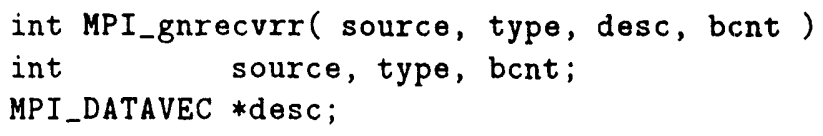

\section{Location}

$$
\text { mpig.c }
$$

MPI_knsend -... Nonblocking send with arbitrary gather

\section{Input Parameters}

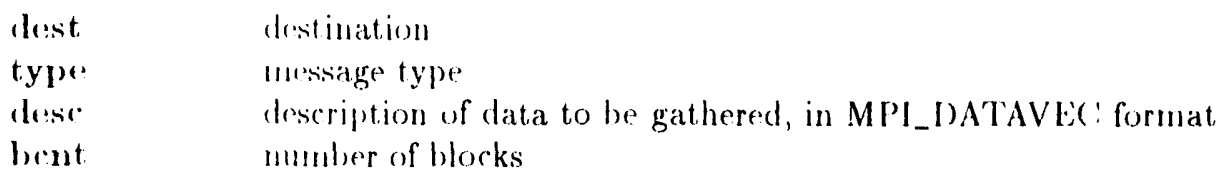

Returns

Integer id of send to be used in MPI_wait, etc., or - 1 on error.

\section{Synopsis}

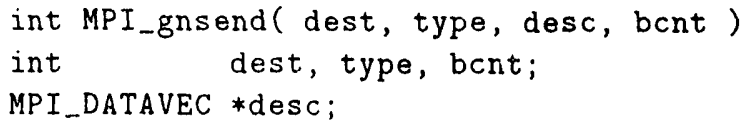




\section{Location}

mpig.c

MPI_gnsendh

MPI_mnsendh

MPI_rnsendh … Noublocking heterogeneous send with arbitrary gather

\section{Input Parameters}

dest

destination

type:

message type

dess:

description of data to be gathered, in MPI_DA'TAVE(: format.

brint number of blocks

\section{Returns}

Integer id of send to be used in MPI_wait, etc., or - 1 on error.

\section{Synopsis}

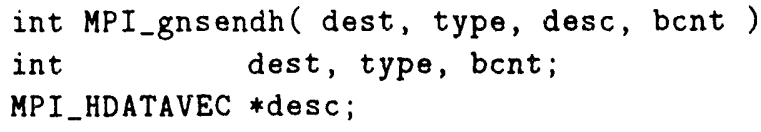

\section{Location}

$$
\text { mpig.c }
$$

MI'I_nnsendhrr - Nonllorking heterogeneous send with arbitrary gather for ready receivers

\section{Input Parameters}

$\begin{array}{ll}\text { dest } & \text { destination } \\ \text { type } & \text { message type } \\ \text { dese } & \text { description of data to be gathered, in MPI_IAATAVEC: format } \\ \text { bent } & \text { number of blocks }\end{array}$

\section{Returns}

Integer id of send to be lused in MPl_wait, etc., or - 1 on error. 
Synopsis

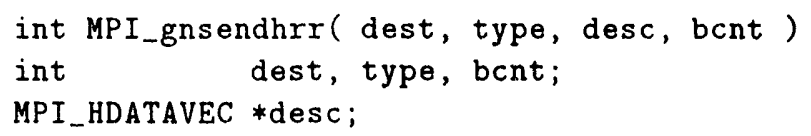

Location

mpig.c

MPI_gnsendrr

MPI_gnsendrr -- Nonblocking send with arbitrary gather for ready receivers

\section{Input Parameters}

$\begin{array}{ll}\text { source } & \text { sending processor } \\ \text { type } & \text { message type } \\ \text { desc: } & \text { description of data to be gathered, in MPI_DATAVEC format } \\ \text { bent } & \text { mumber of blocks }\end{array}$

\section{Returns}

Integer id of send to be used in MPI_wait, etc., or -1 on error.

\section{Synopsis}

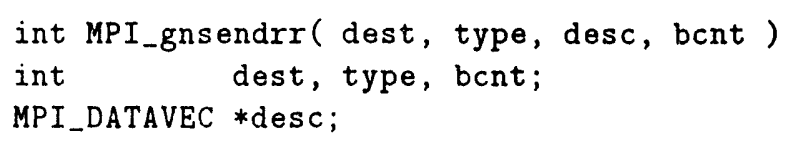

\section{Location}

mpig.c

\subsection{Process Groups}

MPI_child

MPI_child -. (iet information alout the children of a specified group

Input Parameters

$\begin{array}{ll}\text { gid } & \text { process group id } \\ \text { maxlis } & \text { size of clist }\end{array}$




\section{Output Parameter}

clist array containing process group id

\section{Returns}

Number of children in the group, or -1 on error.

Synopsis

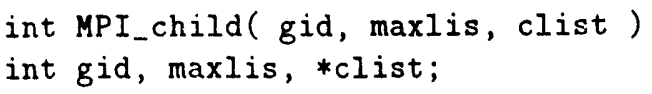

Location

group.c

MPI_copyg - ('reate a root group from an existing group)

\section{Input Parameter}

gid process group id

\section{Returns}

The gid of the new root group, or -1 on error.

Synopsis

int MPI_copyg( gid )
int gid;

\section{Location}

group.c

MPI_defrg

MPI_defrg

MPI_defrg -- Define a root group

\section{Input Parameters}

uprocs number of processes in the group

plist array of PID that are members of the group 


\section{Returns}

The gid of the new root gromp, or - 1 on error.

\section{Synopsis}

$$
\begin{aligned}
& \text { int MPI_defrg( nprocs, plist) } \\
& \text { int nprocs, *plist; }
\end{aligned}
$$

\section{Location}

$$
\text { group.c }
$$

MPI_freed

MPI_freed - Discard the descendants of a specified group

Input Parameter

$$
\text { gid group id }
$$

Returns

0 on success, or -1 on error.

\section{Synopsis}

$$
\text { int MPI_freed( gid ) }
$$$$
\text { int gid; }
$$

Location

$$
\text { group.c }
$$

MPI_freeg -- Discard a specified group and all of its descendents

\section{Input Parameter}

$$
\text { frid group id }
$$

Returns

$U$ on success, or -1 on error. 
Synopsis

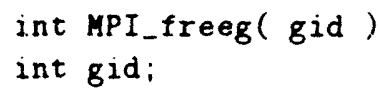

Location

$$
\text { group.c }
$$

MPI_geticl

MPI_getid

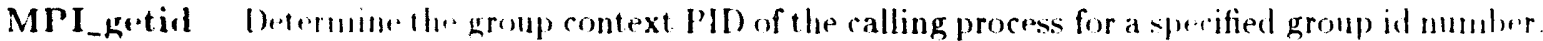

\section{Input Parameter}

$$
\text { gid srump in }
$$

Synopsis

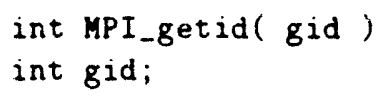

\section{Returns}

Relative process number of the calling process in the specified group.

\section{Location}

mpi.h

MPI_infog

MPI_infogr

MPI_infog Determine the number of processes in a group and return the PII) numbers of the gromp monuluers

\section{Input Parameters}

$$
\begin{array}{ll}
\text { gicl } & \text { kroup id } \\
\text { maxlis } & \text { size of plist } \\
\text { plist } & \text { integer array to hold the members of the group }
\end{array}
$$

\section{Returns}

Hhe mumber if melublers in the group, or - 1 on error. 


\section{Synopsis}

int MPI_infog(gid,maxlis,plist)
int gid, maxlis, *plist;

Location

$\operatorname{mpi}, \mathrm{c}$

MPI_parent

MPI_parent - Determine the group id number of the parent of a specified group

Input Parameter

gid group whose parent is to be found

Returns

The group id of the parent, or -1 on error.

Synopsis

int MPI_parent ( gid )

int gid;

Location

group.c

MPI_partg

MPI_pauth

MPI_parte - Partirion a group into subgroups

\section{Input Parameters}

fid

group to be partitioned

key

key whose value determines the partitioning

\section{Returns}

The gid of the subgronp to which the calling process belongs, otherwise -1 .

\section{Synopsis}

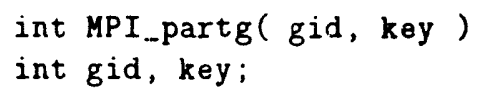




\section{Location}

group.c

MPI_popg

MPI_popr

MPI_popg - Reestablish the process group context.

\section{Returns}

The process group id that is reestablished as the root, otherwise -1 .

Synopsis

int MPI_popg()

Location

group.c

MPI_pusher

MPI_pushg

MPI_pushy - Establish the process group context

\section{Input Parameter}

gid The group context to establish

Returns

The mumber of processes in the group gid, or -1 on error.

Synopsis

int MPI_pushg( gid)

int gid;

Location

group.c

MPI_rooty

MPI_rooter

MPI_roote Ciet information alout root groups 
Input Parameter

maxlis size of rlist

Output Parameter

rlist array of the process group id numbers

\section{Returns}

Number of defined root groups, or -1 on error.

Synopsis

int MPI_rootg ( $\operatorname{maxlis,}$ rlist )

int maxlis, *rlist;

Location

group.c

MPI_sibly

MPI_sibly -- (iet information about the siblings of a group

\section{Input Parameters}

$\begin{array}{ll}\text { gid } & \text { process group id } \\ \text { maxlis } & \text { size of slist }\end{array}$

\section{Output Parameter}

slist array of the proress group ids

\section{Returns}

Number of nillings, or -1 on error.

Synopsis

int MPI_siblg (gid, maxlis, slist)

int gid, maxlis, slist;

\section{Location}

group.c 


\subsection{Communication Contexts}

MPI_infoe:

MPI_infoc

MPI_infor: - (iet information about valid communication contexts

Input parameter

maxlis maxinum size of ilist

Output parameter

ilist array containing communication context ID) numbers

Synopsis

int MPI_infoc( $\operatorname{maxlis,~ilist)}$

int maxlis, *ilist;

Location

mpi.c

MPI_newc.

MPI_newe

MPI_newc..... ('reate a new communication context

\section{Returns}

The id number of a new communication context, or -1 on error.

\section{Synopsis}

int MPI_newc()

\section{Location}

mpi.c

MPI_pope:--.- Reestablish former communication context

\section{Returns}

The id number of a new communication context, or -1 on error. 
Synopsis

$$
\text { int MPI_popc() }
$$

\section{Location}

$$
\text { mpi.c }
$$

MPI_pushe:

MPI_pushe -- Establish a new communication context

\section{Input Parameter}

$$
\text { coid the ID number of communication context to establish }
$$

\section{Returns}

() on success or -1 on error.

Synopsis

$$
\begin{aligned}
& \text { int MPI_pushc( ccid) } \\
& \text { int ccid; }
\end{aligned}
$$

\section{Location}

$$
\text { mpi.c }
$$

\subsection{Program Managemeni}

MPI_main -.. Name of main progran for MPI applications

Note

Use "MPI_main" instead of "main" in (' prograns, and instead of "PROCiRAM <name" in Fortran progranss.

Symopsis

(C)

int MPI_main ( argc, argv)

int arge:

char **argv; 


\section{Synopsis}

(Fortran)

integer function MPI_main()

Location

mpi.c

MPI_stepall

MPI_stopall

MPI_stopall … stop all processes

Input Parameter

re: return code to pass back to calling environment.

Synopsis

void MPI_stopall( rc )

Location

mpi.c

\subsection{Utility Routines}

MPI_crror

MPI_error

MPI_error -- Determine the error status

\section{Returns}

Integer giving the error status for the preceding call to an MPI routine.

Synopsis

int MPI_error()

Location

$\operatorname{mpi} . \mathrm{c}$ 
MPI_etext - Return the text corresponding to an error value

\section{Input Parameter}

ierruo Value returned by MPI_error

Synopsis

char *MPI_etext ( ierrno)

int ierrno;

\section{Location}

mpi.c

MPI_machine -.. (iet machine name, version, and related information

\section{Returns}

A character string giving the name, etc., of the machine.

\section{Synopsis}

$$
\text { char *MPI_machine( ) }
$$

\section{Location}

$$
\operatorname{mpi.c}
$$

MPI_infonm -.. (iet information on the machine configuration

\section{Input Parameter}

$$
\text { maxlis the size of ilist }
$$

\section{Output Parameter}

ilist integer array containing information about the system. 
ilist:[0] - muisher of physical processors in the machine

ilist[1] - total number of processors in the machine

\section{Synopsis}

int MPI_infonm( $\operatorname{maxl}$ is, ilist)

int maxlis, *ilist;

\section{Location}

mpi.c

MPI_syne:

MPI_sync:

MPI_syno -... Synchronize all processes

Synopsis

int sync

Location

$\operatorname{mpi} \cdot \mathrm{h}$ 


\section{Index}

MPl_callcel, (j

MPI_grecuhrr, 38

MPl_cbece, 13

MPl_chreceli, 14

MPlenecevher, 14

MIl_ebrecver, 15

MPl_losend, 15

MPl_cbsendh, 16

MPl_chsendhrr, 16

MPl_chsendrr, 17

MPI_child, 50

MPl_cnrecv, 18

MPl_cnrecvh, 18

MPI_curecuhrr, 19

MPl_curecvrr, 19

MPI_cnsend, 20

MPl_cnsendh, 20

MPl_chsendhre, 2I

IIl'_onsendrr, 21

MPl_copyg, 51

MPl_reev, 8

MPl_recoll, ?

illi_recevher, 9

MPI_crecurr, 10

MPl_rsend, 11

MIl_csendh, 11

MPl_semolher, 12

MPl_csender, 13

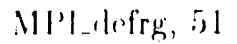

MlPLerwi, 5!

.110. 1.text, 60

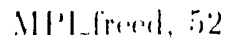

IIfly freeg, 5i2

Mll_glorect 12

IIIfogherwh, 42

Illi-ubrecuher, 43

MPl-sherever, 43

IIPl_olsond, 44

IIPl stsindls. 44

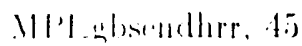

Mll ly bisender, 45

Mll..nentid, 5.3

MPl_zurect, 46

MPl_znrecrlo, 46

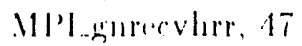

Mlli, merecere, is

Ill'l-ghisemel is

IIPl gusendli, 1!)

.11P/_ntsendher, 19

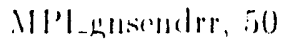

.llPlyeres, 37

III'l-mercuh, 37

MPI_grecvrr, 39

MPI_gsend, 39

MPI_gsendh, 40

MPl_gsendhrr, 40

MPl_gsendirr, 41

MPI_infoc, 57

MPI_infog, 5.3

MPI_infonm, 60

MPI_infos, 6

MPI_infot, 6

MPI_machine, 60

MPI_main, 58

MPI_newc, 57

MPI_parent, 54

MPI_partg, 54

MPI_pope, 57

MPI_popg, 55

MPI_probe, 7

MPI_pushe, 58

MP'I_pushg, 55

MPl_rootg, 55)

MPI_sbrecv, 27

MPI_sbrecvh, 28

MPI_sbrecuhrr, 28

MPI_sbrecvrr, 29)

MPI_sbsend, 30

MPI_sissendh, 30)

MPl_sbsendhre, 31

MPI_sbsendrr, 31

MPl_siblg, 56

MPI_snrect, 32

MPl_surecvh, 33

MPI_surecuhre, 33

MPI_snrecvrr, 34

MPI_snsend, 34

MPl_snsendh, 35

MPl_snsendhrr, 35)

MPI_susendrr, $36^{\circ}$

MPI_srecv, 22

MPI_srecuh, 23

MPI_srecuhrr, 2:3

MPI_srecvrr, 24

MPI_ssend, 25)

MPI_ssendh, 25

MPl_ssendhrr, 26

MPI_ssendrr, 27

MPl_stats, 7

MPl_stopiall, 59

MPI_sync, 61

MPI_wait, 8 
Distribution for ANL-92/47

Internal:
J. M. Beumer (100)
F. Y. Fradin
W. D. Gropp (10)
E. L. Lusk (10)
G. W. Pieper
R. L. Stevens
C. L. Wilkinson
ANL Patent Department
TIS File

External:

DOE-OSTI, for distribution per UC-405 (54)

ANL-E Library

ANL-W Library

Manager, Chicago Field Office, DOE

Mathematics and Computer Science Division Review Committee:

W. W. Bledsoe, University of Texas, Austin

B. L. Buzbee, National Center for Atmospheric Research

J. G. Glimm, State University of New York at Stony Brook

M. T. Heath, University of Illinois at Urbana

E. F. Infante, University of Minnesota

D. O'Leary, University of Maryland

R. E. O'Malley, Rensselaer Polytechnic Institute

M. H. Schultz, Yale University

H. Berryman, Yale University

J. Cavallini, Department of Energy - Energy Research

J. Choi, Oak Ridge National Laboratory

$\mathrm{J}$. Dongarra, University of Tennessee

J. Flower, ParaSoft Corporation

D. Frye, IBM Corporation

A. Geist, Dak Ridge National Laboratory

I. Glendinning, University of Southampton, U.K.

A. Greenberg, Thinking Machines Corp.

L. Hart, National Oceanic and Atmospheric Administration

R. Hempel, GMD, Germany

T. Henderson, National Oceanic and Atmospheric Administration

T. Hey, University of Southampton, U.K.

M. Hill, University of Southampton, U.K.

C. Ho, IBM Almaden Research Center

F. Howes, Department of Energy - Energy Research

S. Huss-Lederman

J. Kapenga, Western Michigan University

K. Kennedy, Rice University

R. Knighten, Intel Scientific Computers

N. MacDonald, University of Edinburgh, U.K. 
o. McBryan, University of Colorado, Boulder

C. Mosher, ARCO Exploration and Production Technology

S. Otto, Oregon Graduate Institute

C. Pancake, Oregon State University

R. Panda, IBM Corporation

M. Peters, IBM UK Scientific Centre

P. Pierce, Intel Scientific Computers

P. Rigsbee, Cray Research, Inc.

A. Skjellum, Mississippi State University

M. Snir, IBM T. J. Watson Research Center

V. Sunderam, Emory University

M. Surridge, University of Southampton, U.K.

L. Tucker, Thinking Machines Corp.

R. van de Geijn, University of Texas, Austin

D. Walker, Dak Ridge National Laboratory

L. Ward, IBM Corporation

S. Zenith, Kuck and Associates, Inc. 

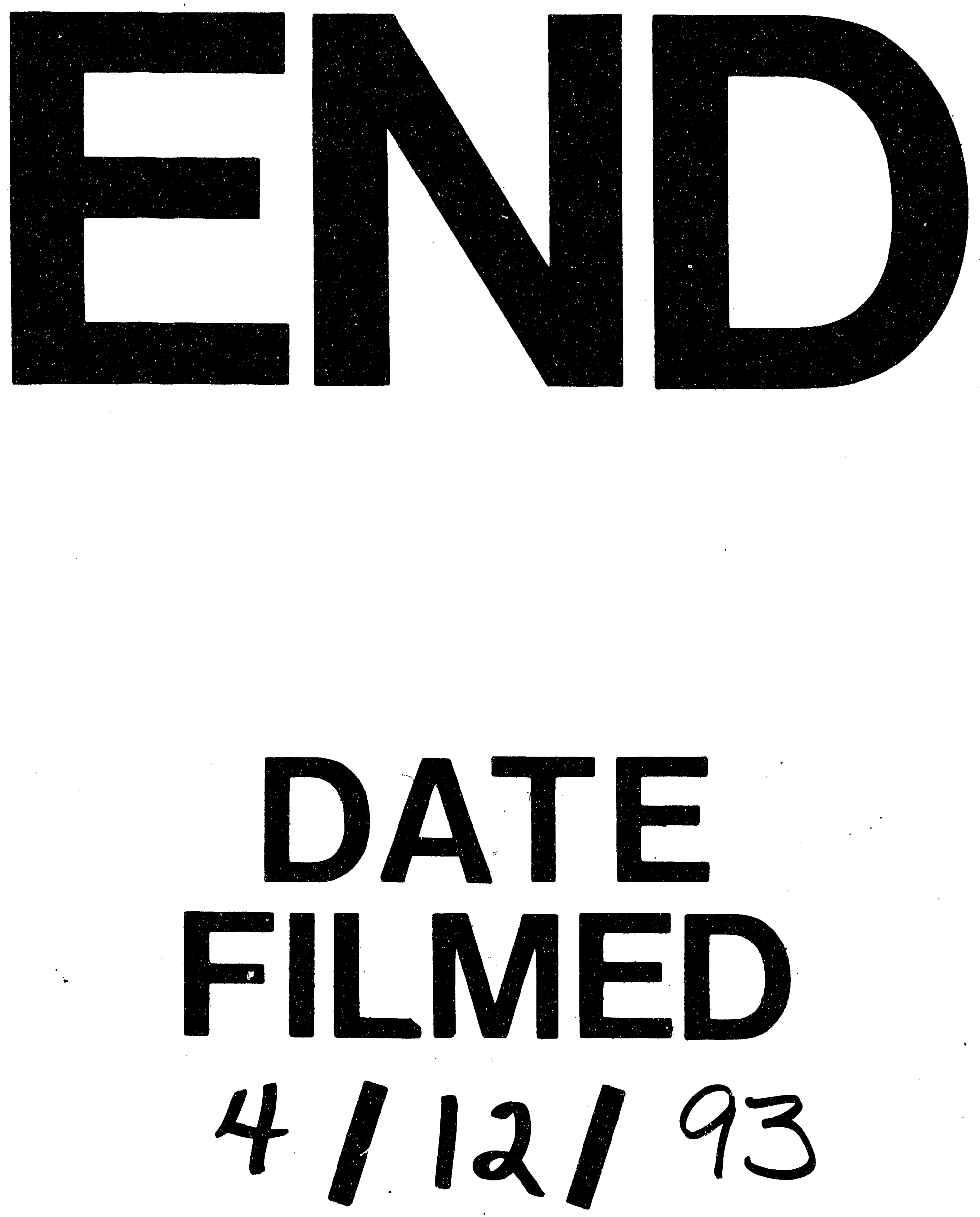
